ARTICLE

Received 5 Feb 2017 | Accepted 15 Mar 2017 | Published 7 Jun 2017

DOl: $10.1038 /$ ncomms15279

OPEN

\title{
The signalling receptor MCAM coordinates apical-basal polarity and planar cell polarity during morphogenesis
}

Qian Gao ${ }^{1,2, \star}$, Junfeng Zhang ${ }^{3, \star}$, Xiumei Wang ${ }^{4}$, Ying Liư ${ }^{4}$, Rongqiao He $\mathrm{H}^{4,5}$, Xingfeng Liu ${ }^{3}$, Fei Wang ${ }^{1}$, Jing Feng ${ }^{1}$, Dongling Yang ${ }^{1}$, Zhaoqing Wang ${ }^{1}$, Anming Meng ${ }^{3} \&$ Xiyun Yan ${ }^{1,2}$

The apical-basal $(A B)$ polarity and planar cell polarity $(P C P)$ provide an animal cell population with different phenotypes during morphogenesis. However, how cells couple these two patterning systems remains unclear. Here we provide in vivo evidence that melanoma cell adhesion molecule (MCAM) coordinates $A B$ polarity-driven lumenogenesis and c-Jun $\mathrm{N}$-terminal kinase (JNK)/PCP-dependent ciliogenesis. We identify that MCAM is an independent receptor of fibroblast growth factor 4 (FGF4), a membrane anchor of phospholipase $\mathrm{C}-\gamma(\mathrm{PLC}-\gamma)$, an immediate upstream receptor of nuclear factor of activated T-cells (NFAT) and a constitutive activator of JNK. We find that MCAM-mediated vesicular trafficking towards FGF4, while generating a priority-grade transcriptional response of NFAT determines lumenogenesis. We demonstrate that MCAM plays indispensable roles in ciliogenesis through activating JNK independently of FGF signals. Furthermore, mcam-deficient zebrafish and Xenopus exhibit a global defect in left-right (LR) asymmetric establishment as a result of morphogenetic failure of their LR organizers. Therefore, MCAM coordination of $A B$ polarity and PCP provides insight into the general mechanisms of morphogenesis.

\footnotetext{
${ }^{1}$ Key Laboratory of Protein and Peptide Pharmaceuticals, Institute of Biophysics, Chinese Academy of Sciences, Beijing 100101, China. ${ }^{2}$ University of Chinese Academy of Sciences, Beijing 100049, China. ${ }^{3}$ State Key Laboratory of Biomembrane and Membrane Engineering, Tsinghua-Peking Center for Life Sciences, Tsinghua University, Beijing 100084, China. ${ }^{4}$ State Key Laboratory of Brain and Cognitive Science, Institute of Biophysics, Chinese Academy of Sciences, Beijing 100101, China. ${ }^{5}$ Key Laboratory of Mental Health, Institute of Psychology, Chinese Academy of Sciences, Beijing 100101, China. ${ }^{\star}$ These authors contributed equally to this work. Correspondence and requests for materials should be addressed to Z.W. (email: clairezqwang@hotmail.com) or to A.M. (email: mengam@mail.tsinghua.edu.cn) or to X.Y. (email: yanxy@ibp.ac.cn).
} 
$\mathrm{D}$ uring morphogenesis, apical-basal (AB) polarity and planar cell polarity (PCP) are prerequisites for organizing cells into accurate structures such as tissues and organs ${ }^{1,2}$. These two distinct patterning systems are composed of two different sets of proteins, but both concurrently operate during morphogenesis ${ }^{3-5}$. However, the major question in morphogenesis, how cells couple these two polarity systems to simultaneously develop different morphologies, remains unclear ${ }^{1,2,5}$.

Formation of multicellular rosette-like structures is a common intermediate process during morphogenesis in diverse species and is a well-studied example of cellular polarity-based processes ${ }^{6,7}$. The zebrafish LR organizer of Kupffer's vesicle (KV) and the posterior lateral line (PLL) system are typical rosette structures ${ }^{5}$. Like adult cells in vertebrate, rosette cells also have ubiquitous organelles of cilia. Each rosette-like structure arises from the proliferation of a group of ciliated cells to form a spherical architecture of polarized cells enclosing a central lumen ${ }^{5}$. Lumenogenesis requires $\mathrm{AB}$ polarity to dictate correct cellular arrangements ${ }^{8}$ and ciliogenesis needs JNK/PCP to regulate cilia assembly ${ }^{4}$.

MCAM, also called CD146 or MUC18, is a signalling receptor and exerts its physiological functions primarily on embryonic development ${ }^{9}$. However, our understanding of how MCAM regulates morphogenesis remains poorly understood. It has been well documented that the polarized distribution of MCAM on the leading edge of chemotaxing cells is vital for establishing cell polarity, but the underlying mechanisms are unclear ${ }^{10}$. In these polarized cells, the concentration of calcium ions $\left(\mathrm{Ca}^{2+}\right)$ is spatially enhanced and polarized in the MCAM-enriched region ${ }^{11} . \mathrm{Ca}^{2+}$ is a crucial signalling element for activation of NFAT, a $\mathrm{Ca}^{2+}$-dependent transcriptional response required for vertebrate development ${ }^{12}$.

FGF signalling plays indispensable roles in the development of living organisms and primarily activates transcriptional factors for activator protein 1 (AP-1), forkhead box protein (FOXO) and $\mathrm{NFAT}^{13}$. The chief intracellular substrates downstream from FGF signalling are FGFR substrate 2 (FRS2) and PLC- $\gamma$. Upon FGF signals, FRS2 switches on AP-1 and FOXO, while PLC- $\gamma$ turns on NFAT. The functional parallels between MCAM and FGF signalling in diverse processes, such as neural patterning, stem cell maintenance, angiogenesis, wound healing and epithelial-mesenchymal transition (EMT) ${ }^{9,14,15}$, hint that an unknown interplay between these two pathways exists.

The zebrafish KV plays central roles in LR asymmetric patterning and is an ideal model for investigation into the epithelial organ morphogenesis of metazoan development ${ }^{16}$. Inspired by the involvement of FGF signalling in the morphogenesis of vertebrate LR organizer and in LR patterning ${ }^{17}$, we hypothesized that MCAM may also exert similar morphogenetic functions as FGF signalling. Here, we have validated this hypothesis at in vitro and in vivo levels. We demonstrate that MCAM coordinates two distinct patterning systems of $\mathrm{AB}$ polarity and $\mathrm{PCP}$, mechanistically explaining how cell populations develop distinct morphologies synchronously. To achieve this coordination, MCAM concurrently activates NFAT and JNK to control $\mathrm{AB}$ polarity and $\mathrm{PCP}$, respectively. Thus, our study suggests that MCAM coordination of $\mathrm{AB}$ polarity and JNK/PCP is a general mechanism for morphogenesis of organs/tissues during development.

\section{Results}

MCAM is an independent receptor of FGF4. MCAM is required for diverse developmental events and is a developmentally regulated signalling receptor with higher abundance in tissues/organs during embryogenesis relative to an adult stage ${ }^{9}$. To decipher the mechanisms underlying MCAM functions in development, we sought to identify MCAM ligands that are necessary and sufficient for morphogenesis. We therefore performed a yeast two-hybrid assay by using the MCAM-GAL4-binding domain fusion protein as the bait to screen a human GAL4-activating domain fusion complementary DNA (cDNA) library.

The transmembrane domain of MCAM was deleted in the constructs to ensure nuclear localization of the MCAM bait proteins. The extra- or intra-cellular domains of MCAM were inserted in-frame into the pGBKT7 expression vector to generate MCAM-1, MCAM-2 and MCAM-3 constructs (Fig. 1a; Supplementary Fig. 1). More than 1,000 prey were sequenced and analysed by BLAST searches, and an array of in-frame coded sequences was identified that represented potential MCAM-interacting proteins. Among them, FGF2, 4 and 8 were identified as prey for MCAM-2 (Fig. 1a).

Endogenous levels of MCAM protein are disparate in different cell lines, with high levels in embryonic or cancerous cells and low levels in most normal cell lines ${ }^{9}$. We screened several cell lines to select one suitable for mimicking morphogenetic processes with high endogenous MCAM. The HEK293 cell line exhibits a high endogenous level of MCAM, comparable to that of several tumour cell types, and was thus selected for use in functional experiments of this investigation (Supplementary Fig. 2).

Co-immunoprecipitation (co-IP) experiments verified that FGF4 and MCAM formed a complex in HEK293 cells (Fig. 1b). Notably, none of the FGFRs (FGFR1-4) tested co-immunoprecipitated with MCAM (Supplementary Fig. 2). FGF4 belongs to the paracrine FGFs that signal FGFRs by forming a tripartite complex with FGFRs and heparan sulphate proteoglycan $(\mathrm{HSPG})^{13}$. To examine the potential requirement of HSPGs for interaction of MCAM with FGF4, we performed co-IP with samples of whole cell lysate that were pre-treated with heparinase to degrade endogenous HSPGs. We found heparinase treatment blocked the association of FGF4-FGFR1 but not that of FGF4-MCAM (Fig. 1b). These data thus provide solid evidence that MCAM is a genuine receptor of FGF4 and transmits FGF4 signals independently of HSPG and separately from FGFRs.

To confirm direct interaction and measure the binding affinity of FGF4 to MCAM, a surface plasmon resonance (SPR) assay was conducted. Results showed that FGF4 bound to MCAM with an affinity of $0.97 \mathrm{nM}$, which was higher than the values observed for FGF4 binding to FGFR1 and FGFR2 (Fig. 1c). FGF2 and 8 did not bind to MCAM although binding to FGFR1 and FGFR2 was detected (Supplementary Fig. 3).

MCAM mediates vesicular transportation in response to FGF4. FGF4 acts as a chemoattractant during the morphogenesis of chick LR organizer ${ }^{18}$. To evaluate the functions of MCAM or FGFRs on cellular behaviours responding to FGF4, we first conducted chemotaxis assays to mimic the process of cell polarity. FGF2 and 8 were included as controls. Post $1 \mathrm{~h}$ addition of FGF2, 4 and 8, MCAM became enriched at the anterior edge of the chemotaxing cells in the direction of the FGF4 gradient. In contrast, FGFR1 was uniformly distributed on the polarized cell membranes in about $80 \%$ of observed cells (Fig. 1d). MCAM enrichment cannot be observed in the chemotaxing cells with the addition of FGF2 and 8 (Supplementary Fig. 4). Furthermore, in about $85 \%$ of chemotaxing cells, MCAM was co-localized with atypical (a) PKC $\zeta$ and PAR3 (two apical markers) at the cellular leading edge in the direction of the FGF4 gradient (Fig. 1e; Supplementary Fig. 5). These results indicate that MCAM is a previously unidentified responsive receptor of FGF4 and that MCAM-enriched leading edge is equivalent to the apical surface of luminal organs. 
a

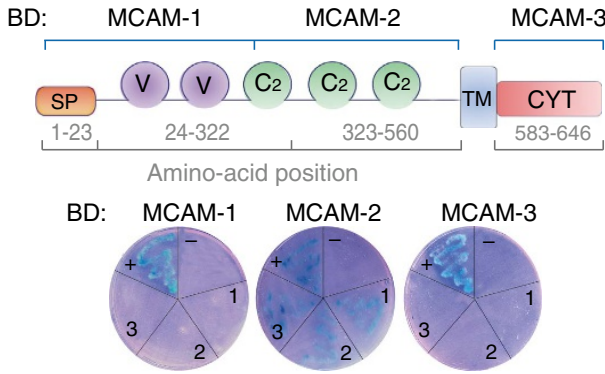

+.BD-mP53 / AD-Vector -. BD-Lam / AD-Vector $\begin{array}{llll}\text { 1. AD-FGF2 } & \text { 2. AD-FGF4 } & \text { 3. AD-FGF8 }\end{array}$ b

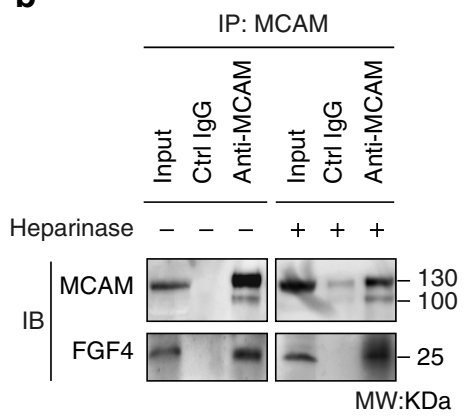

IP: FGFR1

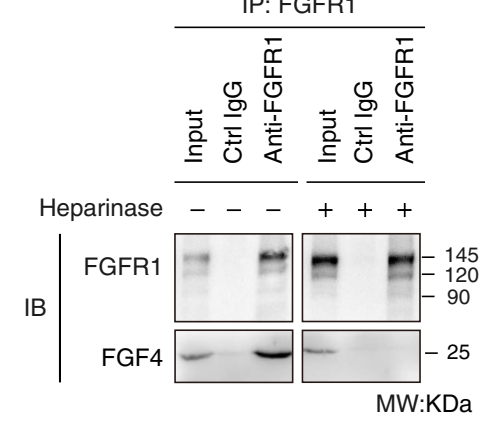

C

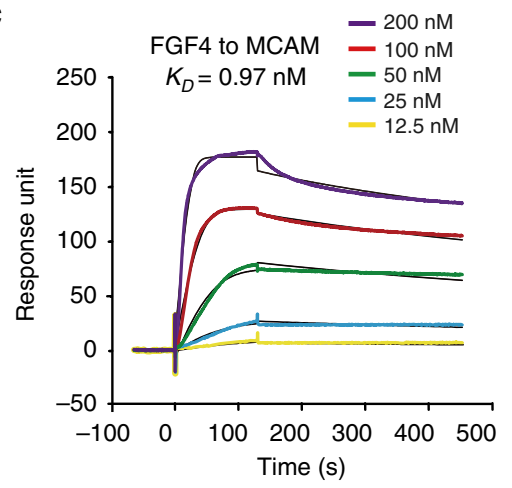

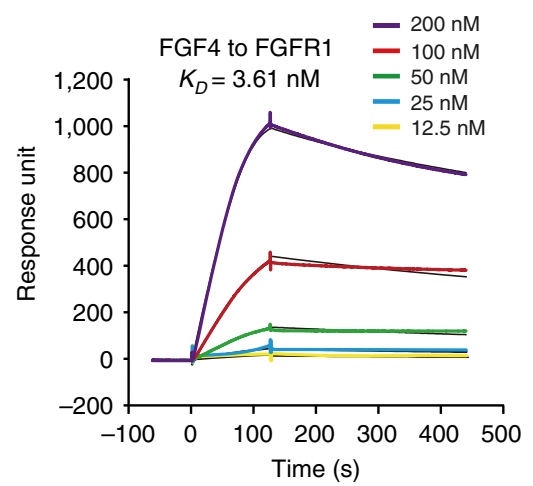

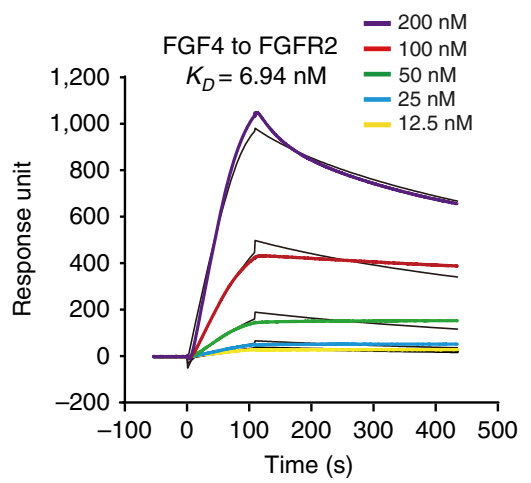

d
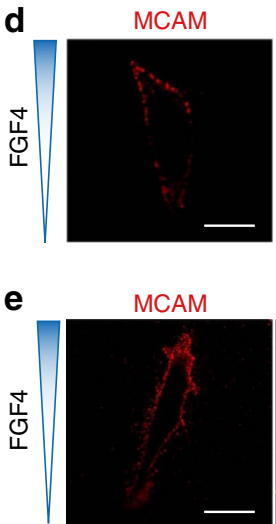

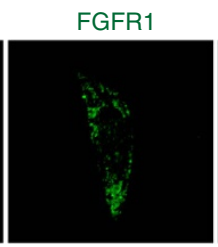

PKC

Nucleus

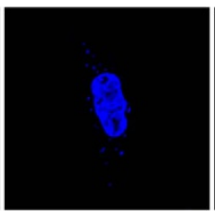

Nucleus

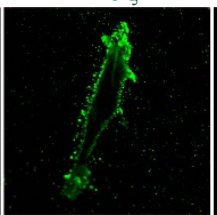

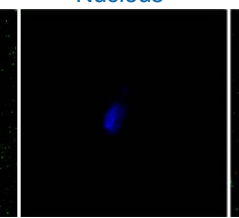

Merged

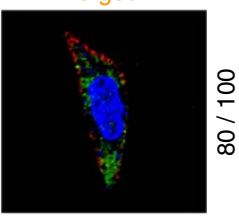

Merged

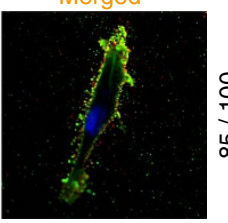

$\mathbf{f}$

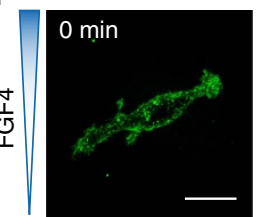

Endogenous MCAM
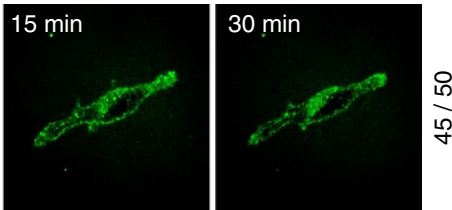

g

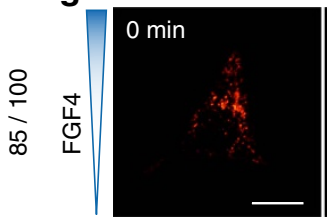

RFP-MCAM

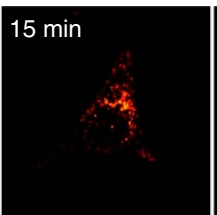

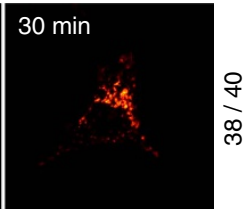

Figure 1 | MCAM/FGF4-dependent apical surface biogenesis. (a) Upper panel, cartoon of MCAM-BD 1-3. Lower panel, yeast zygotes obtained after mating the bait strain containing pGBKT7-MCAM with the library strain containing pGADT7-FGF2, FGF4, and FGF8. BD, DNA-binding domain; $A D$, activation domain; +, positive control with p53-BD; and - , negative control with empty $A D$ vector and BD-lambda. (b) Co-immunoprecipitation of MCAM/FGF4 and FGFR1/FGF4 with the protein lysate treated with or without the heparinase I and heparinase III $\left(0.06 \mathrm{IU} \mathrm{ml}{ }^{-1}\right)$. (c) Kinetic dissociation constant $\left(K_{D}\right)$ of FGF4/MCAM, FGF4/FGFR1, or FGF4/ FGFR2 complexes was measured using a surface plasmon resonance method. (d) Distribution of polarized MCAM and unpolarized FGFR1 on chemotaxing cells. The source concentration of FGF4 in the chemotaxis assay is $10 \mathrm{ng} \mathrm{ml}^{-1}$. (e) Co-localization of endogenous MCAM and the apical marker aPKC $\zeta$ in chemotaxing cells. Scale bar, $20 \mu \mathrm{m}$. (f,g) Time-lapse live-cells imaging of endogenous MCAM (f) or exogenous MCAM-RFP ( $\mathbf{g}$ ) at the leading edge of chemotaxing cells.

To further understand how MCAM enriched at the anterior edge of the chemotaxing cells responds to FGF4, endogenous and exogenous MCAM and endogenous FGFR1 were tracked by live-cell imaging in a chemotaxis assay. After the addition of FGF4, endogenous MCAM became enriched at the anterior edge of the chemotaxing cells in the direction of the FGF4 gradient after an average time of $30 \mathrm{~min}$ in about $90 \%$ of tested cells (Fig. 1f; Supplementary Movie 1). Exogenous MCAM-RFP showed similar localization and trafficking patterns within $30 \mathrm{~min}$ in about $95 \%$ of tested cells. The direction of $\mathrm{MCAM}^{+}$ endocytic vesicular transportation was identical to that of cellular movement towards the FGF4 gradient within the period of monitoring (Fig. 1g; Supplementary Movie 2). In contrast, such movement was not observed for endogenous FGFR1 (Supplementary Movie 3). These results demonstrate that the asymmetric distribution of MCAM is mediated through endocytic vesicular transportation.

Loss of MCAM function impairs lumenogenesis. The core mechanisms underlying the biogenesis of luminal apical surfaces involve the asymmetric distribution of plasma membrane proteins through vesicular trafficking ${ }^{19,20}$. We thus speculated that MCAM is involved in lumenogenesis. To test this hypothesis, we decided to use a three-dimensional (3D) in vitro model to mimic the lumenogenesis process from a single HEK293 cell ${ }^{21}$. 

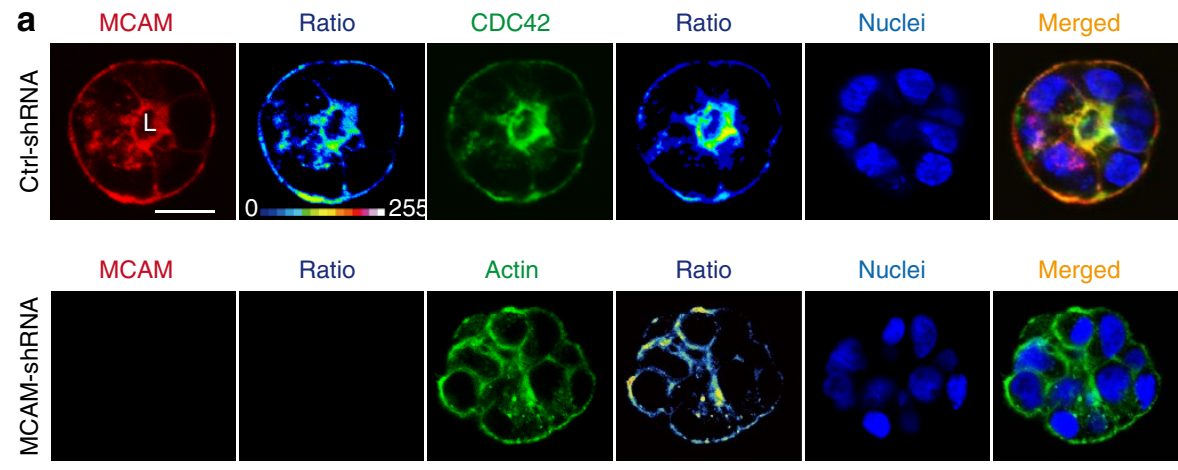

e
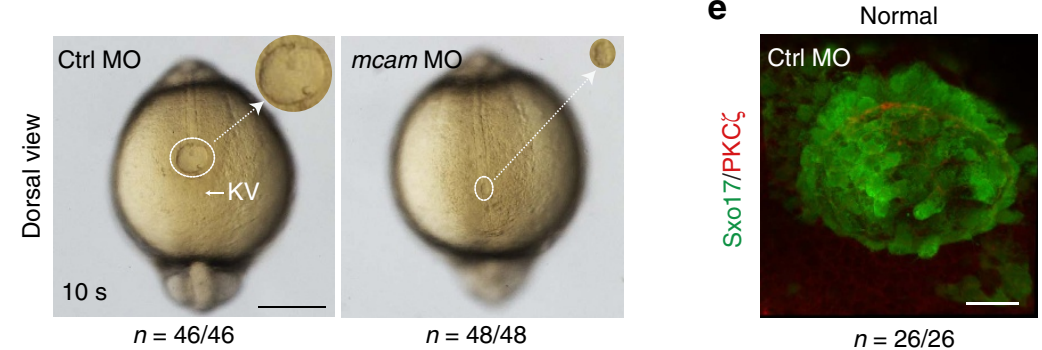

$n=26 / 26$

f

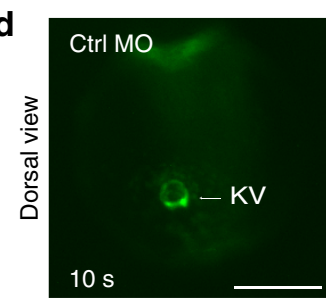

$n=46 / 46$

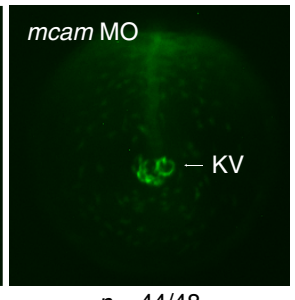

$n=44 / 48$

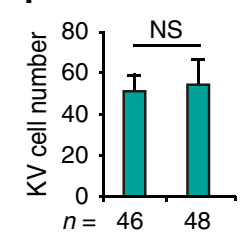

Ctrl MO - + g

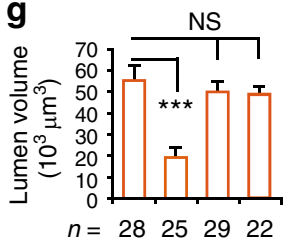

mcam $\mathrm{MO}-+-$

Fgf4 MO --+-
Fgfr1MO $-~$
DIC

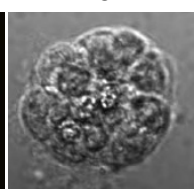

DIC

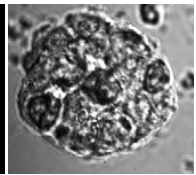

b

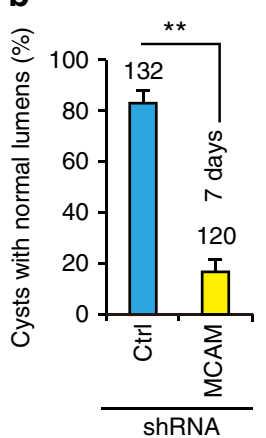

Disrupted

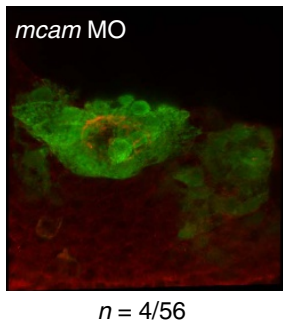

h

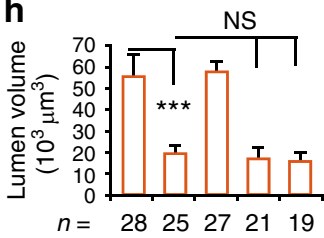

mсат $\mathrm{MO}-++++$

mcam RNA - -+-

Fgf4 RNA $--+\overline{-}-$

Fgfr1 RNA ----+

Figure 2 | Mcam depletion disrupts lumenogenesis. (a,b) HEK293 cells transfected with either control pGPU6/Neo-shRNA vector or pGPU6/ Neo-MCAM-shRNA were cultured on Matrigel for 5-7 days to enable cyst formation. Cyst nuclei and F-actin were labelled with DAPI and phalloidin, respectively. DIC means digital image of contrast. The fluorescent images were converted to 16-colour intensity images indicating the localization of MCAM, CDC42, or F-actin (calibration bar: signal intensity). L, lumen space; Scale bar, $20 \mu \mathrm{m}$. Data (mean \pm s.e.m., $n=3$ ) were analysed with unpaired Student's $t$-test. ${ }^{*} P$ value $<0.01$. (c,d) KV in Sox17:GFP zebrafish TG embryos injected with mcam MO or control MO into DFC. Embryos were collected at $10 \mathrm{~s}$ (somite) stage. In representative light micrographs (c), the area with KV was enlarged 4 times and shown as inset in the right hand corner. Scale bar, $250 \mu \mathrm{m}$. (e) mcam MO was injected into DFC of Sox17:GFP transgenic embryos. Lumen cells were immunolabeled with aPKC antibody (red). Representative fluorescence images show Sox17-GFP-labelled KV (green) and lumen cells (red). Scale bar, $20 \mu \mathrm{m}$. (f) KV cell numbers in DFCmcam MO embryos.

Data (mean \pm s.e.m., $n=3$ ) were analysed with unpaired Student's $t$-test and NS, not significant. (g) Comparison of KV lumen volumes in embryos with DFC injection of mcam, fgf4 or fgfr $1 \mathrm{MO}$. KV lumen volumes were calculated using the measure stack tool of Image J software. Data are presented as mean \pm s.e.m. $(n=3)$. One-way analysis of variance (ANOVA) with Tukey's post-test. ${ }^{\star \star \star} P$ value $<0.001$ and NS, not significant. (h) Rescue effects of mcam, fgf 4 or fgfr1 mRNA on reduction of lumen volumes in DFC mcam MO embryos. Data are presented as mean \pm s.e.m. $(n=3)$. One-way ANOVA with Tukey's post-test. ${ }^{\star \star \star} P$ value $<0.001$ and NS, not significant.

When cells transfected with MCAM-short hairpin (sh) RNA or control shRNA were plated to form cysts (Supplementary Fig. 6), most cells with control shRNA (85\%) could form normal lumens. MCAM was localized at the apical surface of cyst lumens; its distribution pattern was similar with CDC42, a required protein in biogenesis of apical surfaces ${ }^{22}$ and an apical marker for cyst lumens $^{23}$. In contrast, MCAM-shRNA knockdown produced obvious defects in luminal biogenesis, with misshapen lumens and disorganized F-actin (an apical marker for cyst lumens) distribution observed in approximately $83 \%$ of cysts (Fig. 2a,b). These data indicate that MCAM is a crucial element for de novo synthesis of luminal tissues/organs.

Then, we questioned whether FGF4/MCAM signalling plays in vivo roles in morphogenesis. We first chose the zebrafish system as an in vivo model because of its high re-productivity and transparent embryos. There are two mcam genes in zebrafish, mcama and mcamb. Mcamb was the gene examined since it possesses high sequence similarity with the homologous genes in mammals ${ }^{24-26}$. We initially analysed mcam mRNA distribution in zebrafish embryos by whole-mount in situ hybridization (WISH).

Results showed that mcam expression started around mid-gastrulation and was highly enriched in dorsal forerunner cells (DFCs) at $90 \%$ epiboly and bud stages, and in KV from the 1somite stage to the 10 -somite stages. At $21 \mathrm{~h}$ post-fertilization (hpf), mcam was expressed in the blood vasculature, heart, somites and eye field (Supplementary Fig. 7). The spatiotemporal distribution of $f g f 4$ mRNA in DFC and KV was similar with that of mcam, but differed from that of $f g r r 1$ (Supplementary Fig. 8), implying that MCAM is a rational partner of FGF4 for KV development.

$\mathrm{KV}$ morphogenesis includes ciliogenesis and alignment of ciliated cells into luminal structures ${ }^{27}$. To evaluate the specific roles of the investigated genes on $\mathrm{KV}$ morphogenesis, we 
a
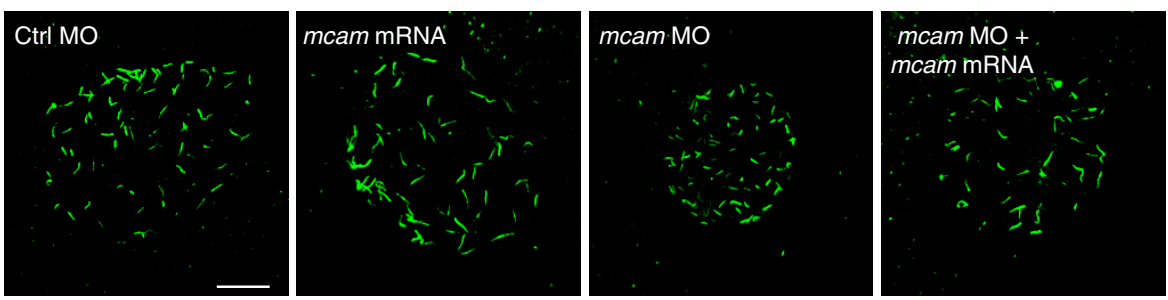

C

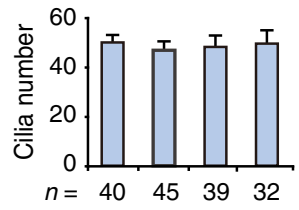

mсат $\mathrm{MO}-+-+$ mcam RNA - -++

g

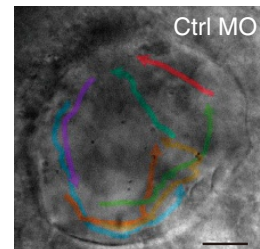

$n=16 / 16$ d

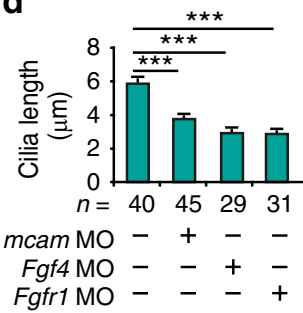

e

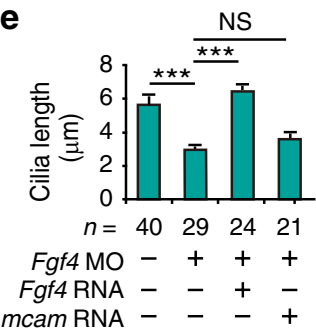

b

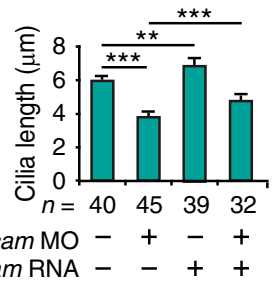

f

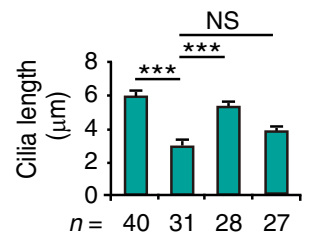

Fgfr1 MO -+++

Fgfr1 RNA - -+-

mcam RNA $-\quad-\quad+$

Figure 3 | Mcam depletion in DFC disrupts ciliogenesis and direction of KV fluid flow in zebrafish. (a) KV cilia labelled with an antibody against acetylated tubulin after DFC injection of embryos with control or mcam MO, mcam mRNA or a combination of mcam MO and mRNA. Scale bar, $20 \mu \mathrm{m}$. (b) Mean cilia length in (a) is statistically analysed. Data are presented as mean \pm s.e.m. One-way ANOVA with Tukey's post-test. ${ }^{\star \star} P$ value $<0.01$ and ${ }^{\star \star \star} P$ value $<0.001$. (c) Mean cilia number (mean \pm s.e.m.) of embryos in a was determined. (d) Comparison of cilia length in embryos with DFC injection of mcam, fgf 4 or fgfr1 MO. Data are presented as mean \pm s.e.m. One-way ANOVA with Tukey's post-test. ${ }^{\star \star \star} P$ value $<0.001$. (e,f) Failure of $m c a m$ mRNA to rescue fgf4 MO (e) or fgfr1 MO (f) induced reduction in cilia length. Data are presented as mean \pm s.e.m $(n=3)$ and analysed using one-way ANOVA with Tukey's post-test. ${ }^{* \star} P$ value $<0.001$ and NS, not significant. (g) Direction of KV fluid flow tracked with fluorescent beads in control and DFC ${ }^{m c a m ~ M O}$ embryos. Scale bar, $20 \mu \mathrm{m}$. (h) Randomized expression of left side-specific spaw in DFC mcam MO morphants. Scale bar, $250 \mu \mathrm{m}$. (i) Quantitative analysis of embryos with normal (left-sided), reversed (right-sided), cardia bifida (bilateral) and absent expression. n, number of embryos (b-f, i).

exclusively knocked-down these genes in DFC/KV, but not in the rest of the embryo, and generated $\mathrm{DFC}^{\mathrm{MO}}$ or mRNA embryos using the transgenic $\operatorname{Tg}(\operatorname{sox} 17: \mathrm{GFP})$ zebrafish line $\mathrm{e}^{28}$. In this line, the $\mathrm{KV}$ can be readily visualized during development owing to the expression of green fluorescent protein (GFP) driven by the promoter of the KV marker Sox17 (ref. 29). Specific knockdown of mcam in DFC/KV caused major morphological defects in the size and shape of the KV at the 10-somite stage (Fig. 2c,d). Labelling the $\mathrm{KV}$ lumen with an antibody against atypical protein kinase $\mathrm{C}$ (aPKC) confirmed that mcam knockdown caused about a $65 \%$ decrease in $\mathrm{KV}$ luminal volume $\left(5.3 \times 10^{4}\right.$ versus $1.8 \times 10^{4} \mathrm{\mu m}^{3}$; $P<0.001$ ), without affecting the number of KV cells (Fig. $2 \mathrm{e}, \mathrm{f}$ ). In contrast, knockdown of $f g f 4$ or $f g f r 1$ in DFC had no effects on the luminal volume of KV (Fig. $2 \mathrm{~g}$ ). The reduction of luminal volume in DFC ${ }^{\text {mcam MO }}$ embryos was rescued by injecting mcam but not $f g f 4$ or $f g f r 1$ mRNA into DFC (Fig. 2h; Supplementary Fig. 9), ruling out the possible contribution of FGF signalling on MCAMmediated $\mathrm{AB}$ polarity-driven lumenogenesis.

Depletion of mcam inhibits ciliogenesis. Depletion of $f g f 4$ or fgfr 1 causes severe shortening of cilia and the consequent disruption of fluid flow within the $\mathrm{KV}^{17,30-32}$. Next, we found that the length of KV cilia also decreased by $35 \%$ in $\mathrm{DFC}^{\text {mcam MO }}$ embryos relative to DFC ${ }^{\text {control } \mathrm{MO}}$ embryos (3.82 versus $5.85 \mu \mathrm{m}$; $P<0.001)$. This reduction in cilia length was rescued by
DFC injection of mcam mRNA into DFC ${ }^{\text {mcam }} \mathrm{MO}$ embryos. In addition, the cilia length was increased by $15 \%$ in DFC mcam mRNA $^{\text {mat }}$ embryos compared to DFC ${ }^{\text {control }} \mathrm{MO}$ embryos (6.77 versus $5.85 \mu \mathrm{m} ; P=0.003$ ) (Fig. 3a,b). Consistent with the unchanged $\mathrm{KV}$ cell number, these injections did not affect the numbers of cilia (Fig. 3c). The effects of cilia shortening caused by mcam knockdown were comparable to those caused by fgfr 1 or $f g f 4$ depletion (Fig. 3d). DFC injection of mcam mRNA cannot rescue the defects of cilia shortening in $f g f 4$ or $f g f r 1$ DFC-morphants, which was rescued only by DFC-injection of $f g f 4$ or $f g f r 1$ mRNA (Fig. 3e,f). However, Mcam overexpression-induced increase of cilia length in wild-type embryos (Fig. $3 b$ ) was not found in $f g f 4$ or $f g f r 1$ depleted embryos (Fig. $3 \mathrm{e}, \mathrm{f}$ ). Thus, these data suggest that the effects of Mcam on cilia length are not entirely independent of FGF signals.

Depletion of mcam disrupts KV function. Zebrafish KV is a fluid-filled organ and the directional nodal flow in KV plays a central role in controlling LR asymmetric development later on $^{32}$. To observe whether or not Mcam is implicated in $\mathrm{KV}$ flow formation, mcam knockdowns in DFC were conducted. In contrast to the persistent counter-clockwise movement of fluorescent beads in the KV lumen of control morphants, a consistent directional flow was absent in the KV of $\mathrm{DFC}^{\text {mcam MO }}$ embryos (Fig. 3g; Supplementary Movies 4 and 5). 
a

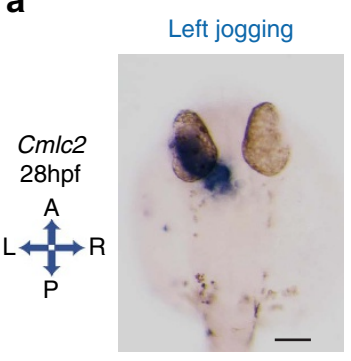

C

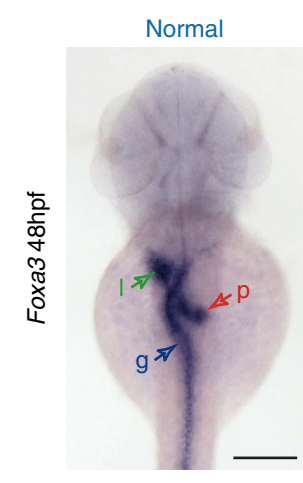

Right jogging
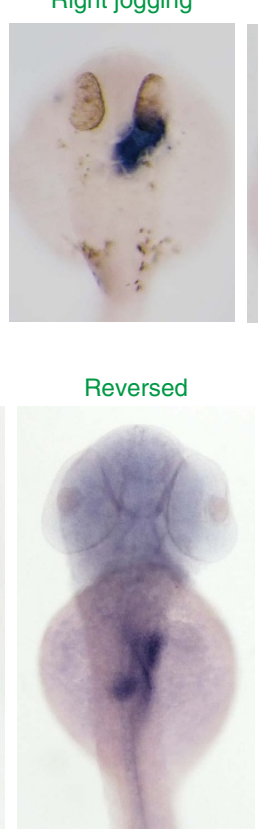

Cardia bifida
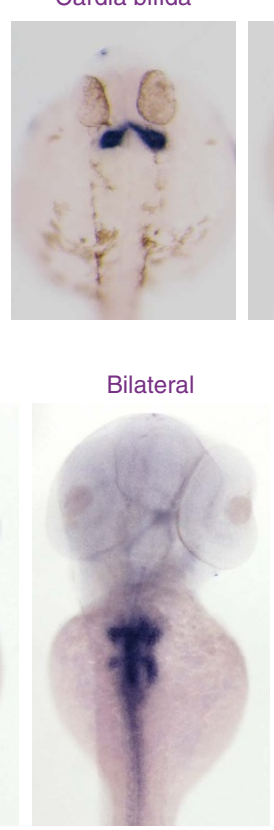

No jogging

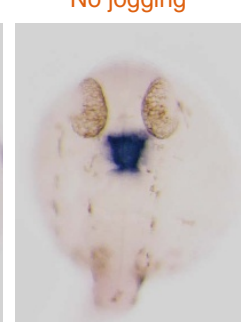

b

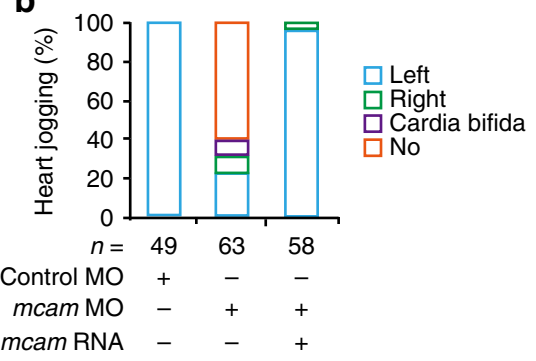

d e

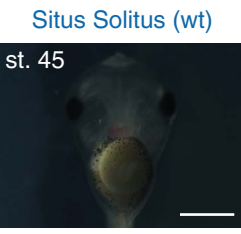

Heterotaxia (ht)

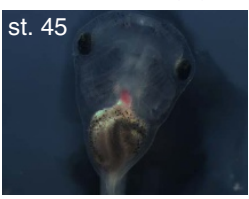

Situs inversus (si)

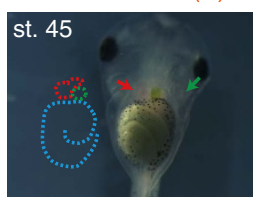

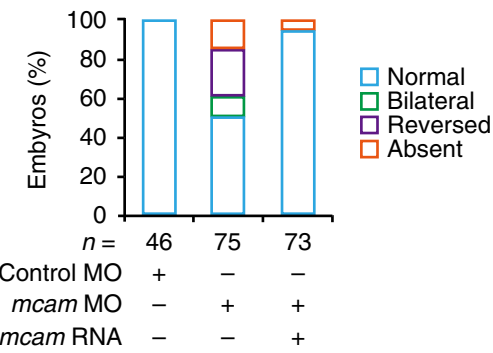

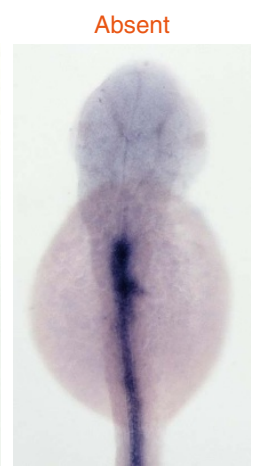

f

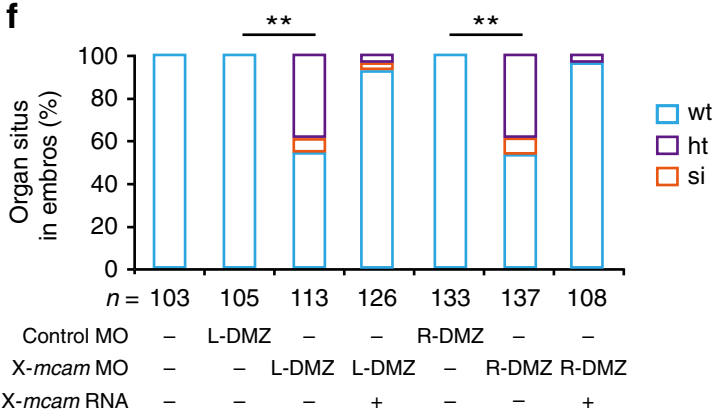

Figure 4 | Mcam regulation of left-right asymmetric development in zebrafish and Xenopus. (a-d) Developing organs in DFC ${ }^{m c a m ~ M O}$ morphants were visualized by WISH using RNA probes of $\mathrm{cmlc2}$ (a, heart) and foxa3 (c, endoderm). Quantitative analysis of embryos with normal, reversed, bilateral and absent asymmetry is shown in $\mathbf{b}, \mathbf{d}$. $n$, number of embryos. The left schema in a shows embryo positions in the embryo ( $A$, anterior; $P$, posterior; $L$, left; and $R$, right). In $\mathbf{c}$, I (green arrow) = liver, $\mathrm{p}$ (red arrow) = pancreas and $\mathrm{g}$ (blue arrow) = gut. H.p.f., hours post-fertilization. Scale bars in a,c , $100 \mu \mathrm{m}$. (e) Xmcam-MO (1 pM) caused situs defects at stage 45, in Xenopus embryos including heterotaxia (ht) and situs inversion (si) compared with the wild type (wt) situs solitus. Heart looping and position is outlined by red dots or red arrow, respectively. Gut coiling is outlined by light blue dots. Position of gall bladder is indicated by green auto-fluorescence and a green arrow. Scale bar, $1 \mathrm{~mm}$. (f) Organ situs in Xenopus embryos at tadpole stage injected as specified. Injections were performed in left or right side of dorsal marginal zone (L- or R-DMZ) at the 4-cell stage. Data are presented as mean \pm s.e.m. Pearson's chi-square tests and ${ }^{\star \star} P$ value $<0.01$. $n$, number of embryos.

Spaw, the earliest left-sided expressed gene, relays nodal flowdelivered signals in KV to initiate a downstream LR signalling cascade in zebrafish ${ }^{31}$. To assess the position of Mcam in the signalling cascade for LR determination, we analysed the expression of spaw and its target genes, including pitx2, lefty1, and lefty 2 in $\mathrm{DFC}^{\text {mcam } \mathrm{MO}}$ embryos. Mcam knockdown in DFC perturbed the normal left-sided expression of spaw and pitx 2 in the lateral plate mesoderm, and induced the randomized expression of lefty 1 and lefty2, the markers for the left part of the dorsal diencephalon and heart primordium, respectively (Fig. 3h,i; Supplementary Fig. 10). These data indicate that, similar to FGF signalling, Mcam is also required at an earlier stage of LR development when the nodal flow in $\mathrm{KV}$ becomes emergence.

Mcam plays conserved roles in LR asymmetry development. $\mathrm{KV}$ is a transient organ to control LR asymmetric development ${ }^{32}$. To observe effects of Mcam in LR determination, the positions of visceral organs were visualized with heart $(\mathrm{cmlc2})$ and liver/pancreas (foxa3) markers by WISH. Mcam deficiency in DFC caused a severe disruption of cardiac jogging, an early feature of cardiac LR asymmetry, as well as a randomization of the heart position (Fig. 4a). Abnormal right jogging (6.7\%), no jogging $(60.0 \%)$ and cardia bifida $(6.8 \%)$ were observed along with normal left jogging (Fig. 4b). LR asymmetry in the liver, gut, and pancreas was also affected by mcam deficiency (Fig. 4c), with the following patterns observed: normal (54.7\%), reversed (22.6\%), bilateral (9.4\%) and absent (13.2\%; Fig. 4d). DFC injection of mcam mRNA can rescue the global defects of LR asymmetry in DFC ${ }^{\text {mcam }} \mathrm{MO}$ embryos (Fig. $4 \mathrm{~b}, \mathrm{~d}$ ).

To explore whether the control of LR asymmetry by MCAM is conserved across vertebrates, we also examined Mcam expression and knockdown effects on organ laterality in Xenopus laevis. Xmcam mRNA was detected by WISH in the gastrocoel roof plate (GRP), the ciliated LR organizer of frogs. Injection of Xmcam-MO into cell lineages of the dorsal marginal zone of 
a

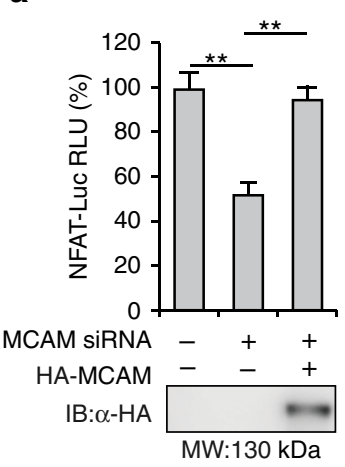

b

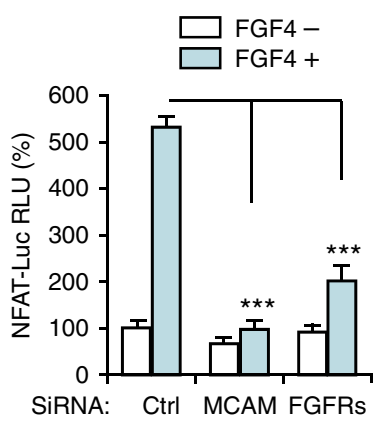

c

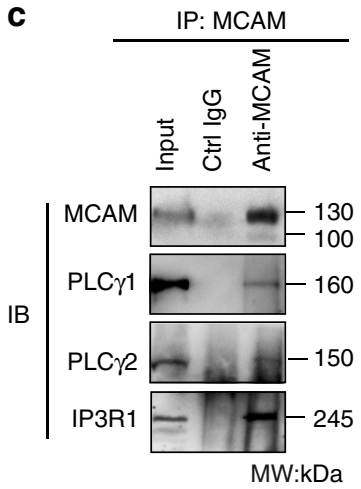

d

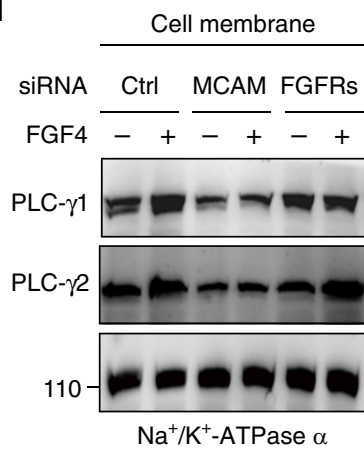

Whole cell

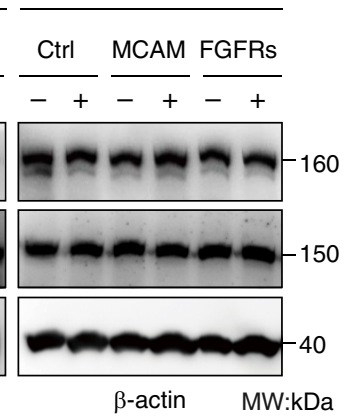

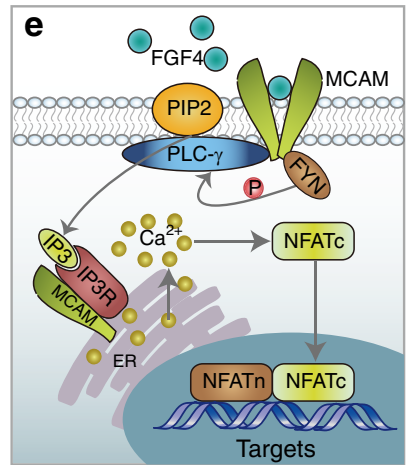

Figure 5 | MCAM controls activation of NFAT. (a) NFAT-Luc reporter assay in HEK293 cells transfected together with MCAM RNAi or MCAM expression vector. RLU means relative luciferase activity. Data are presented as mean \pm s.e.m. $(n=3)$. One-way ANOVA with Tukey's post-test and ${ }^{\star \star} P$ value $<0.01$. (b) After co-transfection of reporter plasmids and RNAi for $20 \mathrm{~h}$, cells were cultured with serum-free medium overnight. NFAT-Luc activity was measured following $1 \mathrm{~h}$ treatment with FGF4 $(2 \mathrm{ng} / \mathrm{ml})$. No treatment served as negative control. Data are presented as mean \pm s.e.m. ( $n=3)$. Two-way ANOVA with Bonferroni post-test and ${ }^{\star \star \star} P$ value $<0.001$. (c) Immunoprecipitation of MCAM followed by immunoblotting (IB). (d) PLC- $\gamma$ abundance in membrane fraction of HEK293 cells and in whole cell lysate after a $48 \mathrm{~h}$ transfection with RNAi with or without treatment with FGF4 for $1 \mathrm{~h}$. (e) Model of MCAM-controlled NFAT activation responding to FGF4. Once dimerization, MCAM conjugates FYN kinase to activate PLC- $\gamma$.

blastomeres, also shortened cilia length relative to control MO-injected embryos (Supplementary Fig. 11). The positions of the gall bladder, heart, and gut looping were inverted (situs inversus) or developed discordantly (heterotaxia) (Fig. 4e,f). These results suggest that MCAM plays conserved roles in LR organizer morphogenesis as well as in LR asymmetric patterning during vertebrate development.

MCAM activates NFAT as an anchor of PLC- $\gamma$. Next, we explored the molecular mechanisms underlying the roles of MCAM in lumenogenesis and ciliogenesis. To identify whether MCAM modulates the PLC- $\gamma$-initiated FGF signalling pathway, the NFAT reporter encoding consensus sequence recognized by a NFAT transcription factor was generated. MCAM knockdown drastically suppressed the activation of NFAT (by $45 \% ; P=0.008$ ) and these reductions were rescued by reconstitution of MCAM (Fig. 5a; Supplementary Fig. 12). There were no direct interactions between MCAM with FRS2 or growth factor receptor-bound protein (Grb) 2, which are critical intermediate signalosomes between activated FGFR and ERK-c-Fos-AP1 or PI3K-PDK-FOXO pathways. The activation of AP-1 or FOXO upon FGF4 stimulation was significantly inhibited in cells with FGFRs knockdown, compared with those cells with MCAM knockdown. In contrast, the effects of MCAM depletion on NFAT activation are higher than those of FGFR1-4 depletion (Fig. 5b; Supplementary Figs 13-15).

In addition, the NFAT upstream elements of PLC- $\gamma 1$, PLC- $\gamma 2$, and $\mathrm{IP}_{3} \mathrm{R}_{1}$ were also identified as candidate MCAM-interacting proteins in the yeast two-hybrid screen. These interactions were further confirmed by co-IP (Fig. 5c), suggesting that MCAM is a component of the $\mathrm{IP}_{3}$ receptor complex and works together with $\mathrm{IP}_{3} \mathrm{R}$ to promote intracellular $\mathrm{Ca}^{2}+$ release. Our findings thus consolidate the previous observations that MCAM resides in the endoplasmic reticulum (ER) and elevates intracellular $\mathrm{Ca}^{2}+$ levels at the MCAM-enriched regions ${ }^{11}$.

Recruitment of PLC- $\gamma$ to the cell membrane is a prerequisite step for the catalysis of membrane-bound $\mathrm{PIP}_{2}$ as well as the subsequent $\mathrm{Ca}^{2}+$ release from $\mathrm{ER}^{30}$. We found that MCAM knockdown impaired PLC- $\gamma 1$ and $-\gamma 2$ anchoring to cell membranes in both the resting and activated status (that is, in the absence or presence of FGF4). By contrast, FGFR1-4 knockdown had no effects on PLC- $\gamma$ recruitment onto cell membranes in either status (Fig. 5d,e). These results, together with previous findings that MCAM recruits Fyn kinase to activate PLC $-\gamma^{33}$, not only demonstrate that MCAM can independently turn on PLC- $\gamma$, but also explain why the knockdown effects of MCAM on NFAT activation are higher than those of FGFRs.

MCAM drives lumenogenesis through NFAT pathway. To evaluate the roles of NFAT activation on morphogenesis, we first blocked NFAT activity in the in vitro 3D cyst cultures with $1 \mu \mathrm{M}$ of 11R-VIVIT (MAGPHPVIVITGPHEE), a specific peptide inhibitor of $\mathrm{NFAT}^{34}$. Statistical analysis indicated that NFAT blockage significantly disrupted lumen formation (Supplementary Fig. 16), which was similar to that of MCAM knockdown (Fig. 2a,b). Thus, these in vitro data 
a

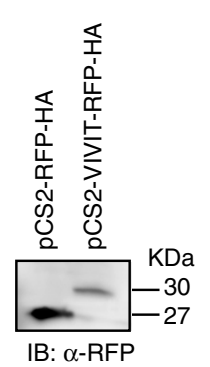

b

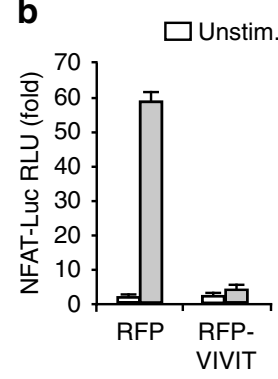

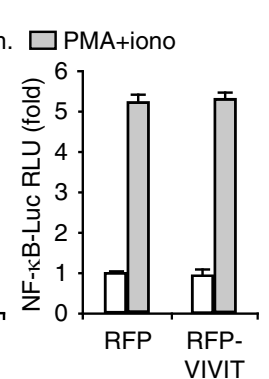

c

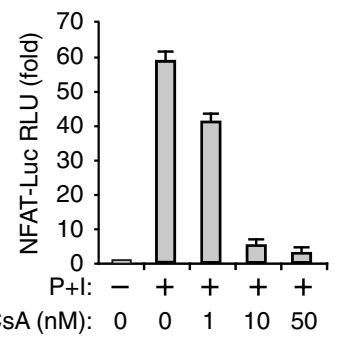

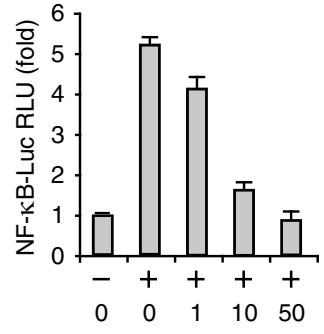
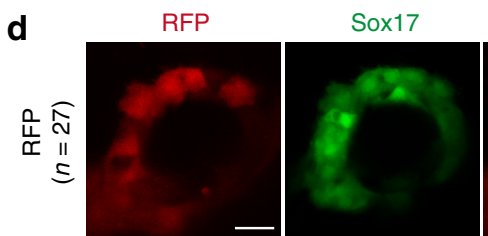

RFP-VIVIT
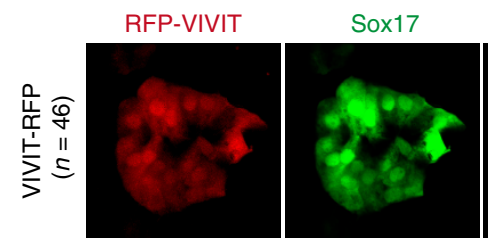

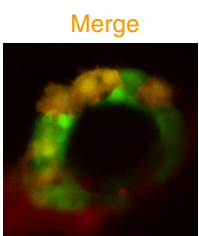

Merge

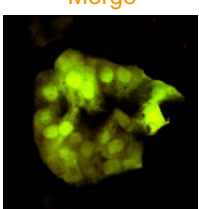

DIC

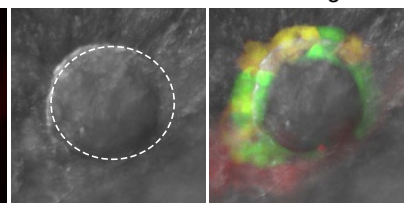

DIC

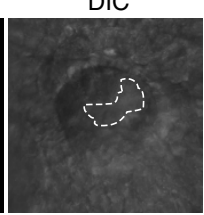

Merged

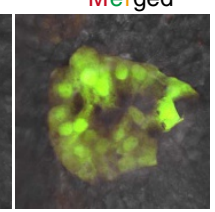

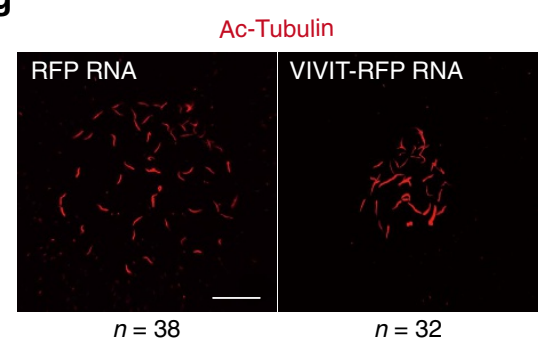

h

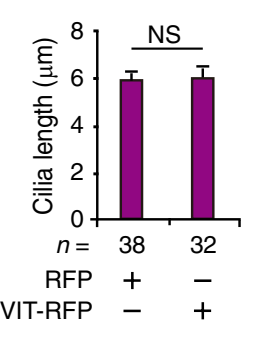

i

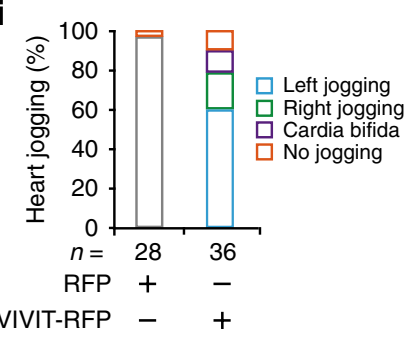

Figure 6 | MCAM controls lumenogenesis by activation of NFAT. (a) Detecting the expression of RFP-HA and VIVIT-RFP-HA in HEK293 cells by IB. (b) VIVIT selectively inhibiting NFAT reporter activity. Jurkat cells were co-transfected with NFAT-Luc (left panel) or NF-kB-Luc (right panel) reporter plasmid, and with RFP and RFP-VIVIT expression plasmids. Twenty-four hours after transfection, cells were left untreated or were stimulated for $6 \mathrm{~h}$ with phorbol 12-myristate 13-acetate (PMA; $20 \mathrm{nM})$ and ionomycin $(1 \mathrm{mM})(\mathrm{P}+\mathrm{I})$. (c) Calcineurin dependence of NFAT and NF-kB reporter activity. Jurkat cells were transfected with NFAT-Luc (left panel) or NF-kB-Luc (right panel) reporter plasmid. Twenty-four hours after transfection, cells were left unstimulated or were stimulated for $6 \mathrm{~h}$ with $\mathrm{P}+\mathrm{I}$ in the absence or presence of $1 \mu \mathrm{M}$ cyclosporin (CsA). (d) Both RFP-tag and VIVIT-RFP were localized and expressed in DFC after microinjection of RFP and RFP-VIVIT mRNA into DFC of zebrafish KV. DIC means digital image of contrast. (e,f) Messenger RNAs were injected into zebrafish DFC of Sox17:GFP transgenic embryos, which were harvested the $10 \mathrm{~s}$ stage. Lumen cells were labelled with an antibody against aPKC $\zeta$ (red). Data are presented as mean \pm s.e.m. One-way ANOVA with Tukey's post-test. ${ }^{\star \star \star} P$ value $<0.001$ and NS $=$ not significant. (g,h) KV cilia were labelled with an antibody against acetylated tubulin after injection of the indicated mRNAs. Data are presented as mean \pm s.e.m. and analysed using unpaired student's t-test. The NS means not significant. (i) Quantitative analysis of heart joggings after injection of the indicated mRNAs into DFC of Sox17:GFP zebrafish embryos. Normal (left), reversed (right) and absent (no) jogging were calculated. Scale bar, $20 \mu$ m. n, number of observed embryos (e-i).

indicate that NFAT is a critical member for $\mathrm{AB}$ polarity-driven lumenogenesis.

To further evaluate the in vivo roles of NFAT in lumenogenesis, we constructed a plasmid containing the VIVIT sequence. The expression of the red fluorescent protein (RFP)-tagged VIVIT blocked activation of an NFAT reporter but not of an NF- $\kappa B$ reporter in Jurkat cells stimulated with phorbol ester (PMA) plus ionomycin (Fig. 6a,b), although both reporters were equivalently sensitive to the inhibition of calcineurin with cyclosporine A (CsA; Fig. 6c).

Similar to observations in DFC ${ }^{\text {mcam MO }}$ embryos (Fig. 2), DFC injection of VIVIT mRNA caused about a $60 \%$ decrease in $\mathrm{KV}$ luminal volume in these zebrafish morphants, as compared to

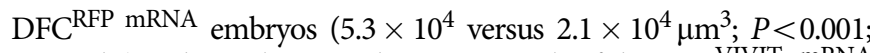
Fig. 6d,e). The volume reduction in zebrafish DFC VIVIT mRNA embryos could not be rescued by injecting DFC with mcam or PLC- $\gamma 1$ mRNA (Fig. 6f). In contrast, blocking NFAT in DFC did not affect cilia length (Fig. 6g,h). Therefore, these in vitro and zebrafish in vivo data demonstrate that NFAT is the effective cascade downstream from the MCAM/PLC $\gamma$ pathway to fulfil lumenogenesis.

Consequentially, blocking NFAT in zebrafish DFC resulted in a severe disruption of cardiac jogging. Abnormal right jogging $(20.1 \%, n=136)$, no jogging $(10.3 \%, n=53)$ and cardia bifida $(10.8 \%, n=30)$ were observed along with normal left jogging in zebrafish embryos (Fig. 6i). The high similarities in defects of 
a

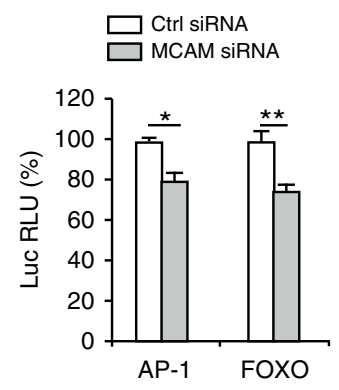

C

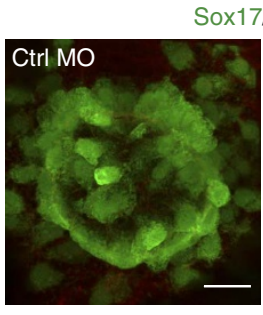

Sox17/PKCל

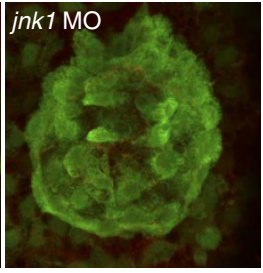

g

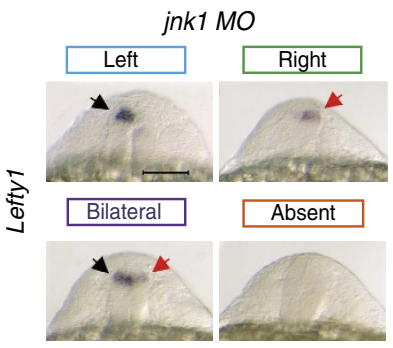

j

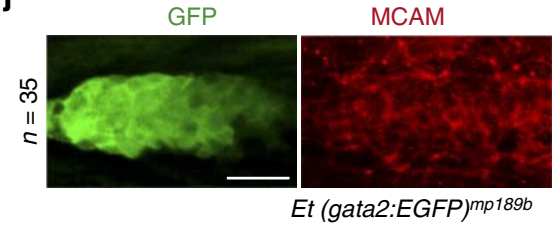

b

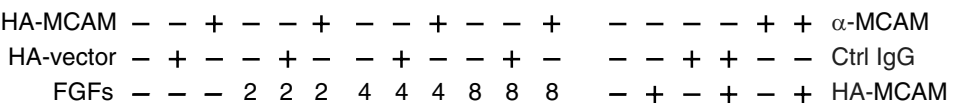
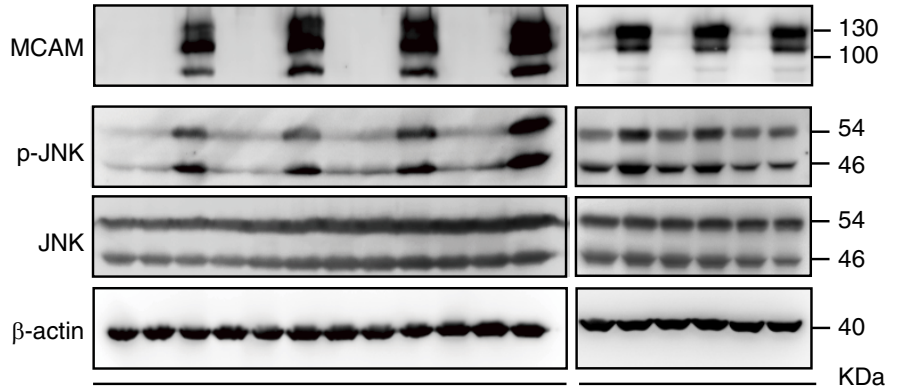

Without serum

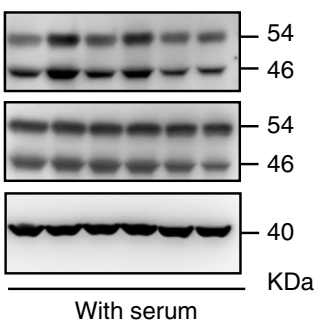

d
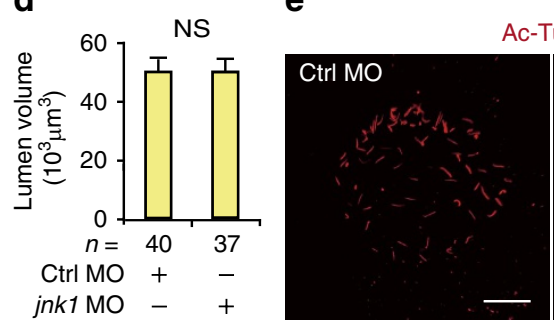

Ac-Tubulin

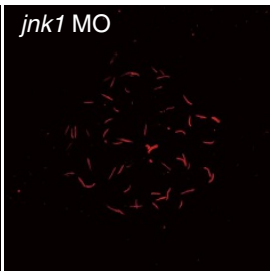

f

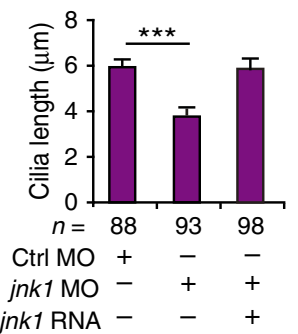

h

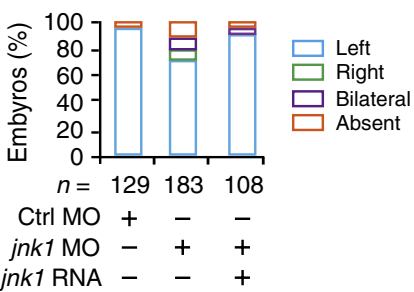

i

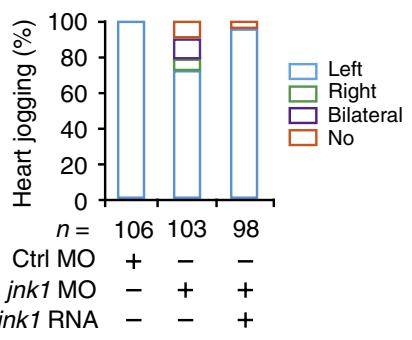

$\mathbf{k}$
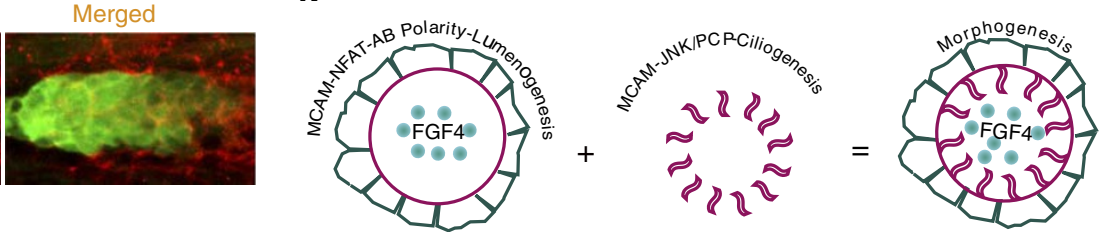

Figure 7 | MCAM-dependent JNK activation regulates ciliogenesis. (a) Luciferase activity measured $30 \mathrm{~h}$ post transfection. AP-1-Luc or FOXO-LuC reporter vectors were co-transfected with either MCAM RNAi or control RNAi. Data (mean \pm s.e.m.) were analysed with unpaired student's $t$-test. ${ }^{\star} P$ value $<0.05$ and ${ }^{* \star} P$ value $<0.01$. (b) Phosphorylation and expression of JNK following overexpression of MCAM by transfection with $p C S 2-M C A M-H A$ or blockage of MCAM by its functional antibody AA98. (c,d) DFC-specific knockdown of jnk1 did not affect lumen formation in Sox17:GFP zebrafish embryos. Lumen cells were labelled with an antibody against aPKC $\zeta$ (red). Scale bar, $20 \mu \mathrm{m}$. Data (mean \pm s.e.m.) were analysed with unpaired student's $t$-test. The NS means not significant. (e,f) DFC-specific knockdown of jnk1 shortened KV cilia. The cilia were labelled with acetylated tubulin antibody. Scale bar, $20 \mu \mathrm{m}$. Data (mean \pm s.e.m.) were analysed using one-way ANOVA with Tukey's post-test. ${ }^{\star \star \star} P$ value $<0.001$. (g,h) Randomized expression of left side-specific lefty1 in the DFC jnk1 MO morphants. Scale bar, $100 \mu \mathrm{m}$. (i) Quantitative analysis of embryos with normal (left-sided), reversed (right-sided), cardia bifida (bilateral) and absent expression. $n$, number of embryos $(\mathbf{d}, \mathbf{f}, \mathbf{h}, \mathbf{I}, \mathbf{j})$. (j) MCAM is localized at the zebrafish PLL system. Embryos of Et (gata2:EGFP) ${ }^{\mathrm{mp} 189 \mathrm{~b}}$ zebrafish line at the 28 h.p.f. stage were collected and stained with MCAM antibody. Scale bar, $15 \mu \mathrm{m}$. (k) A model of MCAMmediated coordination of cell polarity during morphogenesis. See text for details.

luminal formation resulting from MCAM deficiency and NFAT depletion imply that MCAM may be the immediate upstream regulator of NFAT.

MCAM constitutively activates JNK to determine ciliogenesis. Then we explored which signalling cascade downstream from
MCAM is responsible for ciliogenesis. The MCAM knockdown inhibited reporter's activation of AP-1 (by 25\%; $P=0.045$ ) and FOXO (by $30 \% ; P=0.008$; Fig. 7a). Subsequently, we examined the activation status of the key kinases upstream of these two transcriptional factors. We found that MCAM overexpression induced a robust increase in JNK activity with or without FGF2, 4 or 8 stimulation, which was suppressed by blocking MCAM 
with MCAM's functional antibody of AA98 (Fig. 7b). In contrast, overexpression or blockage of MCAM had no effects on the change of ERK, p38 and PDK phosphorylations. ERK phosphorylation was completely dependent on the presence of FGFs, but not on the abundance of MCAM (Supplementary Fig. 17). These results thus demonstrate that MCAM constitutively activates JNK, independent of FGF signals.

We next asked whether jnk is linked with Mcam-regulated KV morphogenesis or ciliogenesis. WISH data with zebrafish embryos indicated that like mcam, jnk1 mRNA expression appeared to be ubiquitous, including in zebrafish $\mathrm{KV}$ (Supplementary Fig. 18). The KV co-expression of Mcam and JNK manifests the spatial rationality that Mcam can access and activate JNK during KV morphogenesis. Next, we found that the DFC knockdown of $j n k 1$ in zebrafish did not affect KV luminal volume (Fig. $7 \mathrm{c}, \mathrm{d}$ ). Notably, DFC knockdown of $j n k 1$ resulted in a reduction of $\mathrm{KV}$ cilia length by $17 \%$ relative to $\mathrm{DFC}^{\text {control } \mathrm{MO}}$ zebrafish embryos ( 4.82 versus $5.85 \mu \mathrm{m} ; P<0.001$ ). Such reduction was rescued by the DFC injection of $j n k 1$ mRNA into those morphants (Fig. 7e,f). The jnk1 knockdown consistently induced randomized expression of spaw and its target genes, including pitx2, lefty1 and lefty2, and caused the significant defect of LR asymmetry in zebrafish embryos (Fig. $7 \mathrm{~g}-\mathrm{i}$; Supplementary Fig. 19). These results demonstrate that constitutive activation of JNK by MCAM is crucial for the determination of cilia length as well as LR asymmetry.

Polarized rosettes are common intermediates during morphogenesis of diverse organs, such as the zebrafish $\mathrm{KV}$, the vertebrate pancreas, as well as the neural stem cell niche. We then asked whether or not the functions of Mcam in morphogenesis are only limited to $\mathrm{KV}$ rosette formation. Beyond $\mathrm{KV}$, the zebrafish PLL system is another widely used model to investigate the cell polarity-driven morphogenesis. Next, we used the transgenic zebrafish Et (gata2:EGFP) mp189b line to trace the development of the PLL system ${ }^{35}$. Labelling endogenous Mcam with immunostaining in this line demonstrates that Mcam is also localized at the PLL system (Fig. 7j and Supplementary Fig. 20), suggesting that MCAM-mediated coordination of $\mathrm{AB}$ polarity and PCP is a broadly utilized mechanism during morphogenesis (Fig. 7k).

\section{Discussion}

A major challenge in understanding morphogenesis is to decipher mechanisms underlying the coordination of $\mathrm{AB}$ polarity and $\mathrm{PCP}$ using genuine tissues. Here we reveal how MCAM simultaneously couples these two distinct patterning systems for lumen formation and cilium growth at the molecular, cellular and in vivo levels. Thus, this study sheds light on the mechanisms by which cell populations synchronously develop distinct morphologies during organ formations.

The unsolved central question in comprehending mechanisms of $\mathrm{AB}$ polarity is to decode the nature of in vivo polarity cues ${ }^{1,36}$. In this study, at both molecular and cellular levels, we demonstrate that FGF4 acts as a spatial cue for biogenesis of apical surface. Thus, in light of this study, morphogens with chemoattractant activity may have the potential to serve as in vivo spatial cues for the establishment of $\mathrm{AB}$ polarity. Furthermore, the cooperation of MCAM/FGF4 in $\mathrm{AB}$ polarity suggests that MCAM could be crucial in FGF4-executed morphogenetic events.

Another open question in cell polarity is to identify the critical polarity complexes that regulate polarized endocytosis ${ }^{1}$. The machinery of endocytic transportation is composed of secretory organelles, including the ER, Golgi complex and endosome. Our findings that MCAM interacts with $\mathrm{IP}_{3} \mathrm{R}_{1}$ of the ER, and mediates endocytic vesicular transportation strongly suggest that
MCAM is a crucial player in the apical polarization of membrane proteins.

Although it has been well established that $\mathrm{PIP}_{2}$ plays a predominant role in the generation of intracellular asymmetry and an apical surface, the upstream signals initiating $\mathrm{PIP}_{2}$ asymmetry remain elusive $e^{3,37,38}$. Our findings that MCAM can independently anchor PLC- $\gamma$, which is also anchored onto the cellular membrane by its interaction with $\mathrm{PIP}_{2}$, suggest that MCAM, PLC- $\gamma$, and $\mathrm{PIP}_{2}$ could form a ternary complex. Therefore, such findings suggest the possibility that the apical distribution of MCAM on a cellular surface could contribute to the origination of $\mathrm{PIP}_{2}$ apical asymmetry.

Formation of cellular polarity requires the orchestration of intracellular signalling events ${ }^{39}$. Our study implies that the FGF4-MCAM-NFAT axis represents an essential signalling pathway for $\mathrm{AB}$ polarity as well as lumenogenesis. PLC- $\gamma$ is a shared signalling nexus that acts downstream from more than 100 cell surface receptors ${ }^{40,41}$. In this regard, PLC- $\gamma$-mediated NFAT activation, elicited from signalling receptors other than MCAM, relies on, at least partially, MCAM-mediated PLC- $\gamma$ activation. Thus, MCAM may be the long-sought-after upstream receptor of NFAT that integrates divergent intracellular $\mathrm{Ca}^{2}+$ signals with NFAT-dependent transcriptional response, for lumenogenesis.

It is well established that JNK activation is dependent on outside environmental signals. Thus, our finding that MCAM constitutively activates JNK is a novel scenario, which facilitates the understanding of MCAM as a requirement in JNK/PCPdependent ciliogenesis ${ }^{42}$. Meanwhile, this constitutive activation of JNK by MCAM can also explain why there is increasing evidence linking MCAM to a wide spectrum of JNK-involved morphogenetic processes.

To our knowledge, our findings provide the first in vivo evidence on how two distinct patterning systems are integrated in genuine tissues and will facilitate better understanding of the key molecular and cellular players of morphogenesis. Therefore, this study is expected to shed light on the general mechanism by which cell populations coordinate two distinct cell polarities during morphogenesis.

\section{Methods}

Antibodies. AA1 $(1: 1,000)$ and AA98 (1:1,000), both of which are murine anti-MCAM monoclonal antibodies generated in our laboratory, recognize the first $\mathrm{V}$ domain and the last $\mathrm{C} 2$ domains in the extracellular region of MCAM, respectively. Additional information for all commercial antibodies is as following. Anti-FGF4 (1:1,000, orb39033) antibody was purchased from Biorbyt limited. Anti-HA-Tag (1:5,000, \#3724), anti-Myc-Tag (1:5,000, \#2276), anti-FGFR1 (1:1,000, \#9740), anti-PLC- $\gamma 1$ (1:1,000, \#2822), anti-PLC- $\gamma 2$ (1:1,000, \#3872), anti-JNK (1:1,000, \#9252S), anti-phospho-JNK (1:1,000, \#9251S), anti-ERK (1:1,000, \#9107), anti-phospho-ERK (1:1,000, \#4370), anti-p38 (1:1,000, \#9107), anti-phospho-p38 (1:1,000, \#4511), anti-PDK (1:1,000, \#3061), anti-phospho-PDK $(1: 1,000, \# 5662)$, Alexa Fluor 488 Phalloidin $(1: 2,000,8878 S)$ and the secondary antibodies of donkey anti-rabbit Alexa Fluor $555(1: 2,000,8953 \mathrm{~S})$ antibodies were from Cell Signaling Technology. Anti-FGF3 (1:2,000, ab10830), anti-FGF8 (1:2,000, ab181492), anti-CDC42 (1:500, ab187643) and anti-PKC $(1: 2,000$, ab119291) antibodies were from Abcam. Anti-FGFR2 (1:2,000, LS-C97522) and anti-IP3R1 (1:2,000, LS-C71062) were from Lifespan. Anti-PAR3 antibody (1:2,000, 07-330) was from Millipore. Anti-FGF2 (1:2,000, AJ1292A), anti-FGFR3 (1:2,000, AP14841c) and anti-FGFR4 antibodies (1:2,000, N-term, AP7639a-ev) were from Abgent. Anti-FRS2- $\alpha$ (1:200, A-5, sc-17841), anti-Grb2 (1:200, sc-255P), anti-Na ${ }^{+} / \mathrm{K}^{+}$-ATPase- $\alpha$ subunit (1:200, sc-21712), anti-Cdc42 (1:200, sc-87) and anti- $\beta$-actin (1:200, sc-8432) antibodies were from Santa Cruz Biotechnology. Anti-RFP-Tag (1:2,000, KM8082) was from Tianjin Sungene Biotech Co., Ltd. The donkey anti-mouse Alexa Fluor 488 (1:2,000, A11053) was from Invitrogen. The secondary antibody, HRP-conjugated goat anti-mouse $(1: 20,000,31446)$ or rabbit $\operatorname{IgG}(1: 20,000,31431)$, was from Thermo Scientific Pierce.

Reagents. Chemicals such as ionomycin, phorbol 12-myristate 13-acetate, cyclosporine, heparinase I, and heparinase III were from Sigma. All cell culture media were from Gibco and all transfection reagents were from Invitrogen. Protease inhibitor, Protein G PLUS-Agarose, and PhosSTOP (phosphatase 
inhibitor cocktail) were from Roche. The Matchmaker Gold Yeast Two-Hybrid System was purchased from Clontech. The Dual-Luciferase System (E1910) was from Promega. The membrane isolation kit was from Thermo Scientific. Heparan Sulfate Proteoglycan (HSPG, PA565Hu01) was from USCN life Science Inc. Human serum albumin recombinant was from California Bioscience. The recombinant FGF4 (AF-100-31) was from Peprotech, and FGF8a (4745-F8-050) was from R\&D Systems. The recombinant human proteins of FGF2 (10014-HNAE), FGFR1/Fc (10616-H03H), FGFR2/Fc (10824-H03H), MCAM/Fc (10115-H02H), and IgG1 Fc (10702-HNAH) were from Sino Biological Inc.

Cell lines with culture conditions and treatments. All of the cell lines were obtained from ATCC, and were authenticated by single nucleotide polymorphism testing and mycoplasma contamination testing. The HEK293 (human embryonic kidney 293, CRL-1573) cell line and the human liver cancer cell line HepG2 (HB-8065) were maintained in EMEM with 10\% fetal bovine serum (FBS). The human malignant melanoma cell line A375 (CRL-1619) was maintained in DMEM with 10\% FBS. The breast cancer cell line MDA-MB-231 (HTB-26) was maintained in EMEM with $10 \% \mathrm{FBS}$ and $0.1 \mathrm{mM}$ of nonessential amino acid (NEAA). The MCF7 (HTB-22) breast cancer cell line was maintained in EMEM with 5\% FBS, $0.1 \mathrm{mM}$ NEAA and $1 \mathrm{mM}$ Sodium Pyruvate. The human colon cancer cell line HT-29 (HTB-38) was maintained in McCoy's 5a medium modified with 10\% FBS. The Jurkat cells (TIB-152) were maintained in a RPMI-1640 medium.

The following are the primers for construction of MCAM-RFP: forward, $5^{\prime}$-gaa ttctgATGGGGCTTCCCAGGCTGGTCT-3' and reverse, $5^{\prime}$-ggatcccgCT AATGCCTCAGATCGATGTAT- 3 '. EcoRI and BamHI were the selected enzymatic sites for cloning procedures. Treatment of sections with $0.006 \mathrm{IU} \mathrm{ml}^{-1}$ heparinase I and heparinase III for $2 \mathrm{~h}$ at $37^{\circ} \mathrm{C}$, followed by the addition of fresh enzyme for an additional $2 \mathrm{~h}$, completely removed endogenous HSPGs ${ }^{43}$.

Yeast two-hybrid screen. The Matchmaker Gold yeast two-hybrid system (Clontech) was used according to the manufacturers' instructions. The yeast strain Y2HGold, which expresses bait protein from the plasmids of pGBKT7-MCAM-1, -2 or -3 , was used to screen the pre-made universal human (normalized) cDNA library (Clontech) in the Y187 yeast strain using a yeast two-hybrid method. Coding sequences of the human FGF2, FGF4, FGF8, PLC- $\gamma 1$, PLC- $\gamma 2$ or $\mathrm{IP}_{3} \mathrm{R}_{1}$ cDNAs were screened from the human cDNA library and confirmed by the sequencing method. The primers for construction of pGBKT7-MCAM-1 (AA, 24322), pGBKT7-MCAM-2 (AA, 323-560) and pGBKT7-MCAM-3 (AA, 583-646) are listed as follows (lower cases indicate added adaptor sequences). MCAM-1 forward, $5^{\prime}$-gccgaattcATGGTGCCCGGAGAGGCTGAGC- $3^{\prime}$; and MCAM- 1 reverse, $5^{\prime}$-ggatccCCTACTGACATTCATAGCGCCCAC- $3^{\prime}$. MCAM-2 forward, $5^{\prime}$-gccgaattcATGGGCCTGGACTTGGACACCA- $3^{\prime}$; and MCAM-2 reverse, $5^{\prime}$-ggatcccCTAGCCCCGGCTCTCCGGCTCC- $3^{\prime}$. MCAM-3 forward, $5^{\prime}$-gccgaat tcATGAAGAAGGGCAAGCTGCCGT- $3^{\prime}$; and MCAM-3 reverse, $5^{\prime}$-ggatcccCTAA TGCCTCAGATCGATGTAT-3'.

Surface plasmon resonance measurements. The binding kinetics between the soluble FGF2, FGF4 or FGF8 to MCAM, FGFR1 or FGFR2, were analysed at room temperature on a Biacore T100 machine (GE Healthcare) with CM5 chips (GE Healthcare) according to a published protocol as following ${ }^{44}$. The buffer of $1 \times$ PBS with $0.05 \%$ Tween 20 was used for all measurements. For surface plasmon resonance (SPR) measurements, two chips were used. Recombinant human chimera proteins of MCAM/Fc, FGFR1/Fc, FGFR2/Fc or IgG1 Fc, were immobilized on the CM5 chip. IgG1 Fc and PBS with $0.05 \%$ Tween 20 were used either as the negative chimera-protein or the blank solvent control. SPR chips were treated using the same protocol; immobilization was conducted using $\mathrm{N}$-hydroxysuccinimide and $\mathrm{N}$-ethyl- $\mathrm{N}$-(dimethylaminopropyl) carbodimide, and neutralization was carried out with $1.0 \mathrm{M}$ ethanolamine. Upon completion of data collection after each cycle, the sensor surface was regenerated by washing with $10 \mathrm{mM}$ glycine- $\mathrm{HCl}$. A series of concentrations were used for the designed experiments. Kinetic and equilibrium parameter $\left(K_{\mathrm{D}}\right)$ values were calculated by fitting the raw sensor gram with the 1:1 binding model using the Biacore T100 evaluation software.

Co-immunoprecipitation and immunoblotting. The HEK293 cells were collected with $5 \mathrm{mM}$ EDTA in PBS and lysed for $1 \mathrm{~h}$ at $4^{\circ} \mathrm{C}$ in a lysis buffer (containing $10 \mathrm{mM}$ Tris-HCl, $100 \mathrm{mM} \mathrm{NaCl}, 50 \mathrm{mM} \mathrm{NaF}, 2 \mathrm{mM}$ EDTA, $0.5 \mathrm{mM}$ sodium vanadate, $1 \% \mathrm{NP}-40$, a protease inhibitor cocktail and a phosphor-stop cocktail, pH 7.5). After removal of cell debris by centrifugation for $15 \mathrm{~min}$ at $14,000 \mathrm{~g}$, proteins $(\sim 500 \mu \mathrm{g})$ were pre-cleaned using normal mouse IgG and Protein G PLUS-Agarose. The pre-cleaned supernatants were incubated overnight with anti-MCAM antibody AA1 at $4{ }^{\circ} \mathrm{C}$ with rotations. The reaction was further incubated with Protein G PLUS-Agarose for three hours at $4^{\circ} \mathrm{C}$. The immunoprecipitates were rinsed three times with a washing buffer $(1 \mathrm{M} \mathrm{NaCl}, 1 \% \mathrm{NP}-40$, $50 \mathrm{mM}$ Tris- $\mathrm{HCl}, \mathrm{pH}$ 7.5) and analysed through immunoblotting (IB) with the desired antibody. The IB was carried out using SDS-polyacrylamide gel electrophoresis (SDS-PAGE), and polyvinylidene difluoride membranes were visualized by the ECL immunoblot detection system. All uncropped western blots can be found in Supplementary Fig. 21.
Immunofluorescence staining and confocal microscopy. Cells were cultured on $\mu$-Slide Ibidi chambers. After $24 \mathrm{~h}$, cells were exposed to a FGF4 gradient with $10 \mathrm{n} \mathrm{ml}^{-1}$ of source, ensuring that the final FGF4 gradient ranges from $0 \sim 4 \mathrm{ng} \mathrm{ml}^{-1}$. Post $2 \mathrm{~h}$ of FGF4-gradient exposure, chemotaxing cells were washed and fixed with $4 \%$ paraformaldehyde in PBS for $25 \mathrm{~min}$. Cells were then permeabilized by treating them with PBS/0.1\% Triton X-100 for $5 \mathrm{~min}$, and then blocked by incubation with 3\% BSA in PBS for $1 \mathrm{~h}$. Cells were then incubated with primary antibodies against MCAM (1:100), FGFR1 (1:50), aPKC (1:100), and PAR3 (1:100) in PBS, with 3\% BSA overnight at $4{ }^{\circ} \mathrm{C}$. Cells were washed three times with PBS/0.1\% Tween 20 and then incubated for one hour with fluorescenceconjugated secondary antibody at a dilution of 1:300 at $37^{\circ} \mathrm{C}$. After $45 \mathrm{~min}$, the cells were washed with PBS/0.1\% Tween 20 and then counterstained with DAPI for DNA. A confocal laser scanning microscope (Olympus FLUOVIEW FV1000) with a mounted Olympus IX81 digital camera was used for image acquisition.

Chemotaxis assay and live-cell imaging. Chemotaxis experiments were carried out in $\mu$-Slide Ibidi chambers according to the manufacturer's instructions, with modifications as described earlier ${ }^{45}$. A HEK 293 cell suspension was diluted to $3 \times 10^{6}$ cells per ml. Six microliter of cell suspension was applied onto one of the filling ports of the $\mu$-Slide using a $20 \mu$ pipettor, and $6 \mu \mathrm{l}$ of air was aspirated from the opposite filling port. The slides were then placed in a sterile $10 \mathrm{~cm}$ Petri dish with a wet tissue around the slide and were transferred to a $37^{\circ} \mathrm{C}$ incubator. After the cells attached, all plugs were gently removed from the filling ports and both reservoirs were filled with $45 \mu \mathrm{l}$ of chemoattractant-free medium. For

chemoattractant application, one of the filling ports was filled with $18 \mu$ l of FGF4 $\left(10 \mathrm{ng} \mathrm{ml}^{-1}\right)$ source solution by removing $18 \mu \mathrm{l}$ of chemoattractant-free medium from the other port on the same side of the device. All the ports were then closed with plugs. Cell migration was recorded by mounting the $\mu$-Slide onto the stage of an inverted microscope (Eclipse Ti, Nikon), which was fitted with a $37^{\circ} \mathrm{C}$ incubator $\left(5 \% \mathrm{CO}_{2}\right)$. Movies were taken using the CSU-X1 spinning disk confocal scanner (UltraVIEW VoX, Perkin-Elmer), a $\times 60$ lens (Nikon), and the Volocity 6.0 software. The movies were taken every minute for RFP-MCAM, endogenous MCAM, or endogenous FGFR1.

Growth of cysts in a three-dimensional matrigel. Cyst cultures in the matrigel were carried out according to an established protocol as following ${ }^{38}$. The matrigel was first thawed at $4{ }^{\circ} \mathrm{C}$ overnight, and then the dishes (Nest, with $15 \mathrm{~mm}$ diameter) were coated with $120 \mu \mathrm{l}$ of Matrigel. The culture dishes were incubated for $15-30 \mathrm{~min}$ at $37^{\circ} \mathrm{C}$ to allow the Matrigel to gel. A single cell suspension with $4 \times 10^{4}$ cells per $\mathrm{ml}$ in $2 \%$ Matrigel was made. The 1,200 $\mu \mathrm{l}$ of cell suspension was plated onto the Matrigel-coated surface. The cultures were maintained for 5-7 days, replacing the Matrigel-medium mixture every two days until cysts with lumen formed. The nuclei of cysts were counterstained with DAPI $\left(0.5 \mu \mathrm{g} \mathrm{ml}^{-1}\right.$ in PBS) for $5 \mathrm{~min}$ at room temperature. Photos were taken with a confocal laser scanning microscope (Olympus FLUOVIEW FV1000) with an Olympus IX81 digital camera.

Fish handling. The zebrafish lines of Tubingen strain, $\mathrm{Tg}$ (sox17: GFP) s870 and Et(gata2a:EGFP)mp189b 35 were maintained following standard protocols. Embryos were raised in an E3 buffer at $26-30^{\circ} \mathrm{C}$. All zebrafish experiments were conducted in an ethical manner as approved by the Animal Care and Use Committee of Tsinghua University. Embryo stages, according to the established criterion $^{46}$, are indicated in the relevant figures and legends. WISH was performed according to standard procedures. For $\mathrm{DFC}^{\mathrm{MO}}$ experiments, a volume of $1 \mathrm{nl}$ fluorescent $\mathrm{MO}$ was injected into embryo yolks at the 500-1,000 cell stage, and embryos were selected by fluorescence microscopy for $\mathrm{MO}$ enrichment as the established standard ${ }^{28}$.

RNA probes of zebrafish. Digoxigenin-labelled RNA probes of zebrafish $\mathrm{mcam}^{24}$, fgf4, fgfrl, foxa3 (ref. 47), cmlc2 (ref. 48), southpaw ${ }^{49}$, pitx2 (ref. 50), lefty1 (ref. 51) and lefty2 (ref. 51) were synthesized by in vitro transcription using T7 or SP6 RNA polymerase.

Antisense morpholino oligonucleotides. Morpholino oligonucleotides (MOs) were synthesized by Gene-Tools, LLC (Corvallis, OR). The sequences of MOs used in the zebrafish were as follows: mcam $\mathrm{MO}^{24}, 5^{\prime}$-AGCAGTGCGGTGTAGGTCA TTTCTC-3'; mcam MO $5^{\prime}$ mis control ${ }^{24}, 5^{\prime}$-AGGCGTGCGGAGTAGCTCATT TGTC-3'; fgf $4 \mathrm{MO}^{52}, 5^{\prime}$-GCTACCGTTTTTCTCTATGCTTGAG-3'; fffr $1 \mathrm{MO}^{17}$, 5'-GCAGCAGCGTGGTCTTCATTATCAT-3'; jnk1 MO (designed based on GenBank sequence AB030900), 5'-ACTGTATCCTGGGCATTCAAGAAG-3'; jnk1 MO $5^{\prime}$ mis control, $5^{\prime}$-ACTGTATGCTCCCGATTGAAGAAG- $3^{\prime}$. The MO of Z-mcam was injected with a dosage of $4 \mathrm{ng}$ for each embryo.

Sequences of morpholinos used in Xenopus laevis were as follows: Xmcam-MO, 5'-ATGAGAGAAGTCATGGTCCTGCTAC-3'; Xmcam MO 5' mis, 5'-ATCA GAGAACTCGTGATCCTGCTTC- $3^{\prime}$. The MO of Xmcam was injected with $15 \mathrm{ng}$ for each embryo. 
Kupffer's vesicle flow analysis. Zebrafish embryos at the 6 to 8 somite stage were dechorionated and mounted in a $1 \%$ low melt agarose. Fluorescent beads $(0.5-2 \mu \mathrm{m}$, Polyscience, Inc) were injected into the KVs and imaged on a Leica DMRA compound microscope, using a camera (UltraVIEW VoX, Perkin-Elmer) with a $\times 40$ lens. Metamorph software (Universal Imaging Corp.) was used to track the injected beads and to calculate bead velocity.

Xenopus laevis handling and RNA probe of Xenopus laevis. Xenopus laevis frogs (Nasco, Atlanta, GA, USA) were stimulated to lay egg by injection of human chorionic gonadotropin. Embryos were obtained by in vitro fertilized and dejellied with $3.2 \mu \mathrm{M}$ DL-Dithiothreito, $\mathrm{pH} 8.9$, and cultured in $0.1 \times 0.1 \times$ Marc's modified Ringer's (MMR; $0.1 \mathrm{M} \mathrm{NaCl}, 20 \mathrm{mM} \mathrm{KCl}, 1 \mathrm{mM} \mathrm{MgSO}_{4}, 2 \mathrm{mM} \mathrm{CaCl}_{2}, 5 \mathrm{mM}$ Hepes, pH 7.8, 0.1 mM EDTA pH8.0 ${ }^{53}$. All animal work received ethics approval from the Animal Care and Use Committee of Institute of Biophysics, Chinese Academy of Sciences.

For Classification of organ situs defects, embryos at stage 45 were anaesthetized in $0.15 \%$ MS222 (Sigma-Aldrich, St Louis, MO, USA), $0.1 \times$ MMR and recorded under stereo microscope (Olympus SZX16, Japan). Situs defects were classified into three types, complete inversion of organ situs (situs inversus), random organ transposition (heterotaxia) and situs solitus (wild type) according to deviation from the normal position and morphology ${ }^{54}$.

Probes for Xenopus laevis mcam and Xnr-1 (ref. 55) were synthesized with linearized pCS2 + HA-Xmcam (a full-length cDNA obtained as described below) and pCS2-Xnr-1 (a $388 \mathrm{bp}$ fragment of the $5^{\prime}$-coding region) as templates, respectively.

Synthesis of mRNAs. In vitro synthesis of mRNAs was performed using the mMessage mMachine kit (Ambion) from the following linearized pCS2 + HA plasmids: Z-mcam forward, 5'-accatgggcATGACCTACACCGCACTGCT-3'; and Z-mcam reverse, $5^{\prime}$-ggatccTTCAGTGGGTGACTTTTTG-3'. Z-fgf4 forward, $5^{\prime}$-accatgggcATGAGTGTCCAGTCGGCCCTC-3'; and Z-fgf4 reverse, $5^{\prime}$-ggatccAA TTCTAGGCAAGAAATGT-3'. Z-fgfr 1 forward, $5^{\prime}$-accatgggcATGATAATGAAG ACCACGCTG-3'; and Z-fgfrl reverse, $5^{\prime}$-ggatccGCGCTTTTTAAAGGCCAC TCCTC-3'. Z-plc 1 forward, $5^{\prime}$-accatgggcATGGCTGCGAGCGCGGGC-3'; and Z- plcy 1 reverse, $5^{\prime}$-ggatccCGCTCGGTTATCGGCGCGAC-3'. Z-jnk1 forward, $5^{\prime}$-accatgggcAGAAACTCAGCCGACCCTT-3'; and Z-jnk1 reverse, 5' -ggatccGCA GCCAATAGACCAGACAT- $3^{\prime}$. Xmcam forward, $5^{\prime}$-accatgggcATGAATTCTCTC ATACTGCTT- $3^{\prime}$; and Xmcam reverse, $5^{\prime}$-ggatccTCAGTTTCTTAAATCCATG TA-3' (gene bank accession number KJ913668.1).

The coding sequence of the RFP-tagged NFAT inhibitor, RFP-VIVIT peptide (MAGPHPVIVITGPHEE), was inserted into the plasmid of pCS2 + HA. The linearized pCS2 + HA-VIVIT-RFP plasmid was used as the template for in vitro synthesis of the RFP-VIVIT mRNA. VIVIT forward 5 -accatgggcATGGCTG GGCCTCATCCCGTC- $3^{\prime}$ and VIVIT reverse $5^{\prime}$-ggatccGGCGCCGGTGGAGT GACGGCCC-3'. The mRNA was injected into zebrafish or Xenopus laevis with a $250 \mathrm{pg}$ dose of mRNA for each embryo.

Gene silencing with siRNA and shRNA. SiRNAs were purchased from GenePharma. The siRNAs against FGFR1, FGFR2, FGFR3 and FGFR4 were designed to target their coding regions. The siRNAs against MCAM were designed to target at its $3^{\prime}$-untranslated region in such way that it results in the specific inhibition of endogenous MCAM RNA, without affecting exogenous transcripts. The concentration of each siRNA is indicated in the respective figures, and the transfection followed the manufacturer's instructions of Lipofectamine RNAi MAX. The siRNA sequences used were as follows: MCAM -\#1 sense is $5^{\prime}$-CAGGUGAAUUAGCCUCAAUTT- $3^{\prime}$. MCAM-\#2 sense is $5^{\prime}$-GGUGUGUAAAUUUGCAAAUTT- $3^{\prime}$. FGFR1-\#1 sense is $5^{\prime}$-GGAGGUGCUUCACU UAAGATT-3'. FGFR1-\#2 is SMART pool (M-003131-02) from Dharmacon (Chicago, IL). FGFR2-\#1 sense is 5'-GGCCUCUCUAUGUCAUAGUTT-3'. FGFR2-\#2 sense is $5^{\prime}$-GGACUUGGUGUCAUGCACCTT-3'.FGFR3-\#1 sense is $5^{\prime}$-GCACACACGACCUGUACAUTT-3'. FGFR3-\#2 sense is $5^{\prime}$-CGACAAGG AGCTAGAGGTT- $3^{\prime}$. FGFR4-\#1 sense is $5^{\prime}$-GCCGACACAAGAACAUCAUTT-3. FGFR4-\#2 sense is $5^{\prime}$-CCACCACAUUGACUACUAUTT- $3^{\prime}$. The plasmid of pGPU6/Neo-MCAM-shRNA was constructed by inserting the antisense sequence of the MCAM-siRNA-\#1.

Luciferase reporter assay. Cells $\left(1 \times 10^{4}\right)$ were transfected with $0.2 \mu \mathrm{g}$ of reporter constructs and $4 \mathrm{ng}$ of pRL-TK (Promega) using Lipofectamine 2,000 reagent (Invitrogen). Cells were transfected with MCAM-siRNA or FGFR1-4-siRNAs, or co-transfected with MCAM-siRNA and HA-tagged MCAM-containing vectors for overexpression of exogenous MCAM. After $24 \mathrm{~h}$ of transfection, cells were treated with or without FGFs as indicated in the respective figures. Both firefly and Renilla luciferase activities were measured using a dual-luciferase reporter assay system in a Glomax multi-detection system luminometer (Promega). Firefly luciferase activity was normalized against Renilla luciferase activity, which was detected by co-transfection with pRL-TK in all reporter experiments. The luciferase activity was normalized against the luciferase activity of cells transfected with the pGL3-E control vector. The relative luciferase activities were expressed as fold increase over the paired control cells. The pGL3-E-NFAT, AP-1, FOXO or NF- $\mathrm{KB}$ plasmids were constructed by inserting consensus sequences to the respective transcription factor binding sites, and are indicated as following: pGL3-E-NFAT ${ }^{56}, 5^{\prime}$-AggAAAA AC-3'; pGL3-E-AP-1 (ref. 57), 5'-ATgAgTCAT-3'; pGL3-E-FOXO ${ }^{58}, 5^{\prime}$-gTA AACA-3'; pGL3-E-NF- $\mathrm{KB}^{59}, 5^{\prime}$-gggACTTTC-3'.

Zebrafish immuostaining. The embryos were collected at desired stages and their chorions were removed. They were then fixed with $4 \%$ paraformaldehyde overnight at $4{ }^{\circ} \mathrm{C}$. The fixed embryos were dehydrated with sequentially graded PBST/ methanol series $\left(3: 1,1: 1\right.$ and 1:3) and $100 \%$ methanol. They were stored at $-20^{\circ} \mathrm{C}$ for more than $30 \mathrm{~min}$. After that, the embryos were rehydrated with sequentially graded methanol/PBST series (3:1, 1:1 and 1:3) and 100\% PBST. The PBST was removed with $1 \mathrm{mM}$ EDTA ( $\mathrm{pH} 8.0$ ) and the embryos were boiled in this EDTA solution for $10 \mathrm{~min}$. They were then cooled slowly to room temperature. The embryos were washed with PBST and PBS-Triton X-100 (0.5\%) sequentially. The embryos were blocked with a $3 \%$ BSA-PBST solution for at least an hour, and then incubated with anti-MCAM $(1: 100)$ overnight at $4{ }^{\circ} \mathrm{C}$. After washing them three times with PBST, the embryos were incubated with the fluorescence labelled secondary antibody $(1: 200)$ overnight at $4{ }^{\circ} \mathrm{C}$. The nuclei were stained with DAPI $\left(1 \mu \mathrm{g} \mathrm{ml}^{-1}\right)$. After washing three times with PBST, the embryos were mounted with coverslips and photographed with a confocal microscope.

Statistical analysis. Statistical analysis was performed in SPSS. Data are shown as mean \pm s.e.m. All data sets were tested for normal distribution with normality tests before proceeding with parametric or non-parametric analysis. Unpaired Student's $t$-test was applied to data sets with normal distributions. When appropriate in case of multiple comparisons, one-way analysis of variance (ANOVA) with Tukey post-test or two-way ANOVA with Bonferroni post-test was applied. Statistical evaluation of experiments represented by bar graphs was performed using Pearson's chi-square tests. A value of $P<0.05$ was considered significant. In experiments of cell confocal microscopy, at least 100 samples were acquired to ensure adequate statistical power. For live-cell imaging experiments, embryo lumenogenesis analysis, and embryo organ patterning, the number of samples was mainly constrained by the complexity of the experimental procedure, data acquisition and analytical capacity. No exclusion criteria were used in experiments. To prevent selection bias, samples of cells and genotype matched pools were randomly divided into experimental groups. Data were analysed automatically where possible to avoid subjective assessments.

Data availability. The DNA sequence of Xenopus mcam has been deposited inGene Bank under the accession code KJ913668.1. The authors declare that all the data supporting the findings of this study are available within the article and its supplementary information files or from the corresponding author upon reasonable request.

\section{References}

1. St Johnston, D. \& Ahringer, J. Cell polarity in eggs and epithelia: parallels and diversity. Cell 141, 757-774 (2010)

2. Zallen, J. A. Planar polarity and tissue morphogenesis. Cell 129, 1051-1063 (2007).

3. Comer, F. I. \& Parent, C. A. Phosphoinositides specify polarity during epithelial organ development. Cell 128, 239-240 (2007).

4. Gray, R. S., Roszko, I. \& Solnica-Krezel, L. Planar cell polarity: coordinating morphogenetic cell behaviors with embryonic polarity. Dev. Cell 21, 120-133 (2011).

5. Harding, M. J., McGraw, H. F. \& Nechiporuk, A. The roles and regulation of multicellular rosette structures during morphogenesis. Development 141, 2549-2558 (2014).

6. Bedzhov, I. \& Zernicka-Goetz, M. Self-organizing properties of mouse pluripotent cells initiate morphogenesis upon implantation. Cell 156, 1032-1044 (2014).

7. Durdu, S. et al. Luminal signalling links cell communication to tissue architecture during organogenesis. Nature 515, 120-124 (2014).

8. Roignot, J., Peng, X. \& Mostov, K. Polarity in mammalian epithelial morphogenesis. Cold Spring Harb. Perspect. Biol. 5, a013789 (2013).

9. Wang, Z. \& Yan, X. CD146, a multi-functional molecule beyond adhesion. Cancer Lett. 330, 150-162 (2013).

10. Witze, E. S., Litman, E. S., Argast, G. M., Moon, R. T. \& Ahn, N. G. Wnt5a control of cell polarity and directional movement by polarized redistribution of adhesion receptors. Science 320, 365-369 (2008).

11. Witze, E. S. et al. Wnt5a directs polarized calcium gradients by recruiting cortical endoplasmic reticulum to the cell trailing edge. Dev. Cell 26, 645-657 (2013).

12. Crabtree, G. R. \& Schreiber, S. L. SnapShot: Ca2 +-calcineurin-NFAT signaling. Cell 138, 210 210.e211 (2009).

13. Goetz, R. \& Mohammadi, M. Exploring mechanisms of FGF signalling through the lens of structural biology. Nat. Rev. Mol. Cell Biol. 14, 166-180 (2013). 
14. Zeng, Q. et al. CD146, an epithelial-mesenchymal transition inducer, is associated with triple-negative breast cancer. Proc. Natl Acad. Sci. USA 109, 1127-1132 (2012).

15. Yan, X. et al. A novel anti-CD146 monoclonal antibody, AA98, inhibits angiogenesis and tumor growth. Blood 102, 184-191 (2003).

16. Capdevila, J., Vogan, K. J., Tabin, C. J. \& Izpisua Belmonte, J. C. Mechanisms of left-right determination in vertebrates. Cell 101, 9-21 (2000).

17. Neugebauer, J. M., Amack, J. D., Peterson, A. G., Bisgrove, B. W. \& Yost, H. J. FGF signalling during embryo development regulates cilia length in diverse epithelia. Nature 458, 651-654 (2009).

18. Yang, X., Dormann, D., Munsterberg, A. E. \& Weijer, C. J. Cell movement patterns during gastrulation in the chick are controlled by positive and negative chemotaxis mediated by FGF4 and FGF8. Dev. Cell 3, 425-437 (2002).

19. Lubarsky, B. \& Krasnow, M. A. Tube morphogenesis: making and shaping biological tubes. Cell 112, 19-28 (2003).

20. Guillot, C. \& Lecuit, T. Mechanics of epithelial tissue homeostasis and morphogenesis. Science 340, 1185-1189 (2013).

21. Lee, G. Y., Kenny, P. A., Lee, E. H. \& Bissell, M. J. Three-dimensional culture models of normal and malignant breast epithelial cells. Nat. Methods 4, 359-365 (2007).

22. Wells, C. D. et al. A Rich1/Amot complex regulates the Cdc42 GTPase and apical-polarity proteins in epithelial cells. Cell 125, 535-548 (2006).

23. O’Brien, L. E. et al. Racl orientates epithelial apical polarity through effects on basolateral laminin assembly. Nat. Cell Biol. 3, 831-838 (2001).

24. Chan, B., Sinha, S., Cho, D., Ramchandran, R. \& Sukhatme, V. P. Critical roles of CD146 in zebrafish vascular development. Dev. Dyn. 232, 232-244 (2005).

25. So, J. H. et al. Gicerin/Cd146 is involved in zebrafish cardiovascular development and tumor angiogenesis. Genes Cells 15, 1099-1110 (2010).

26. Ye, Z. et al. Wnt5a uses CD146 as a receptor to regulate cell motility and convergent extension. Nat. Commun. 4, 2803 (2013)

27. Matsui, T. \& Bessho, Y. Left-right asymmetry in zebrafish. Cell. Mol. Life. Sci. 69, 3069-3077 (2012).

28. Amack, J. D. \& Yost, H. J. The T box transcription factor no tail in ciliated cells controls zebrafish left-right asymmetry. Curr. Biol. 14, 685-690 (2004).

29. Sakaguchi, T., Kikuchi, Y., Kuroiwa, A., Takeda, H. \& Stainier, D. Y. The yolk syncytial layer regulates myocardial migration by influencing extracellular matrix assembly in zebrafish. Development 133, 4063-4072 (2006).

30. Kadamur, G. \& Ross, E. M. Mammalian phospholipase C. Annu. Rev. Physiol. 75, 127-154 (2013)

31. Hamada, H., Meno, C., Watanabe, D. \& Saijoh, Y. Establishment of vertebrate left-right asymmetry. Nat. Rev. Genet. 3, 103-113 (2002).

32. Hirokawa, N., Tanaka, Y., Okada, Y. \& Takeda, S. Nodal flow and the generation of left-right asymmetry. Cell 125, 33-45 (2006)

33. Anfosso, F. et al. Activation of human endothelial cells via S-endo-1 antigen (CD146) stimulates the tyrosine phosphorylation of focal adhesion kinase p125(FAK). J. Biol. Chem. 273, 26852-26856 (1998).

34. Aramburu, J. et al. Affinity-driven peptide selection of an NFAT inhibitor more selective than cyclosporin A. Science 285, 2129-2133 (1999).

35. Sang, Q. et al. Ildr1b is essential for semicircular canal development, migration of the posterior lateral line primordium and hearing ability in zebrafish: implications for a role in the recessive hearing impairment DFNB42. Hum. Mol. Genet. 23, 6201-6211 (2014).

36. Lecuit, T. \& Le Goff, L. Orchestrating size and shape during morphogenesis. Nature 450, 189-192 (2007)

37. Pinal, N. et al. Regulated and polarized PtdIns(3,4,5)P3 accumulation is essential for apical membrane morphogenesis in photoreceptor epithelial cells. Curr. Biol. 16, 140-149 (2006).

38. Martin-Belmonte, F. et al. PTEN-mediated apical segregation of phosphoinositides controls epithelial morphogenesis through Cdc42. Cell 128, 383-397 (2007).

39. Iruela-Arispe, M. L. \& Davis, G. E. Cellular and molecular mechanisms of vascular lumen formation. Dev. Cell 16, 222-231 (2009).

40. Crabtree, G. R. \& Olson, E. N. NFAT signaling: choreographing the social lives of cells. Cell 109(Suppl): S67-S79 (2002).

41. Shaw, J. P. et al. Identification of a putative regulator of early $\mathrm{T}$ cell activation genes. Science 241, 202-205 (1988).

42. Boutros, M., Paricio, N., Strutt, D. I. \& Mlodzik, M. Dishevelled activates JNK and discriminates between JNK pathways in planar polarity and wingless signaling. Cell 94, 109-118 (1998).

43. Allen, B. L., Filla, M. S. \& Rapraeger, A. C. Role of heparan sulfate as a tissue-specific regulator of FGF-4 and FGF receptor recognition. J. Cell Biol. 155, 845-858 (2001).

44. Shi, Y. et al. Structures and receptor binding of hemagglutinins from humaninfecting H7N9 influenza viruses. Science 342, 243-247 (2013).

45. Suraneni, P. et al. The Arp2/3 complex is required for lamellipodia extension and directional fibroblast cell migration. J. Cell Biol. 197, 239-251 (2012).
46. Kimmel, C. B., Ballard, W. W., Kimmel, S. R., Ullmann, B. \& Schilling, T. F. Stages of embryonic development of the zebrafish. Dev. Dyn. 203, 253-310 (1995).

47. Odenthal, J. \& Nusslein-Volhard, C. fork head domain genes in zebrafish. Dev. Genes Evol. 208, 245-258 (1998).

48. Huang, C. J., Tu, C. T., Hsiao, C. D., Hsieh, F. J. \& Tsai, H. J. Germ-line transmission of a myocardium-specific GFP transgene reveals critical regulatory elements in the cardiac myosin light chain 2 promoter of zebrafish. Dev. Dyn. 228, 30-40 (2003).

49. Long, S., Ahmad, N. \& Rebagliati, M. The zebrafish nodal-related gene southpaw is required for visceral and diencephalic left-right asymmetry. Development 130, 2303-2316 (2003).

50. Essner, J. J., Branford, W. W., Zhang, J. \& Yost, H. J. Mesendoderm and leftright brain, heart and gut development are differentially regulated by pitx2 isoforms. Development 127, 1081-1093 (2000).

51. Bisgrove, B. W., Essner, J. J. \& Yost, H. J. Regulation of midline development by antagonism of lefty and nodal signaling. Development 126, 3253-3262 (1999)

52. Yamauchi, H., Miyakawa, N., Miyake, A. \& Itoh, N. Fgf4 is required for left-right patterning of visceral organs in zebrafish. Dev. Biol. 332, 177-185 (2009).

53. Cordenonsi, M. et al. Occludin dephosphorylation in early development of Xenopus laevis. J. Cell Sci. 110(Pt 24): 3131-3139 (1997).

54. Blum, M. et al. Xenopus, an ideal model system to study vertebrate left-right asymmetry. Dev. Dyn. 238, 1215-1225 (2009).

55. Jones, C. M., Kuehn, M. R., Hogan, B. L., Smith, J. C. \& Wright, C. V. Nodal-related signals induce axial mesoderm and dorsalize mesoderm during gastrulation. Development 121, 3651-3662 (1995).

56. Jain, J., Miner, Z. \& Rao, A. Analysis of the preexisting and nuclear forms of nuclear factor of activated T cells. J. Immunol. 151, 837-848 (1993).

57. Rao, A., Luo, C. \& Hogan, P. G. Transcription factors of the NFAT family: regulation and function. Annu. Rev. Immunol. 15, 707-747 (1997).

58. Papadia, S. et al. Synaptic NMDA receptor activity boosts intrinsic antioxidant defenses. Nat. Neurosci. 11, 476-487 (2008).

59. Dixit, V. \& Mak, T. W. NF-kappaB signaling. Many roads lead to madrid. Cell 111, 615-619 (2002).

\section{Acknowledgements}

This work was supported in part by grants from the National Natural Science Foundation of China (81371330, 91529306, and 81272409), National Basic Research Program of China (2015CB553705) and 'Strategic Priority Research Program' of the Chinese Academy of Sciences (XDA12020207).

\section{Author contributions}

Z.W., A.M. and X.Y. designed the study and interpreted the data and wrote the paper with input from all authors. Q.G. and J.Z. performed all the experiments, with the exception of Xenopus laevis handling performed with X.W., Y.L. and R.H. X.L conducted immunostaining of zebrafish. Antibody producing and purification were performed by F.W., D.Y. and J.F.

\section{Additional information}

Supplementary Information accompanies this paper at http://www.nature.com/ naturecommunication

Competing interests: The authors declare no competing financail interests.

Reprints and permission information is available online at http://npg.nature.com/ reprintsandpermissions/

How to cite this article: Gao, Q. et al. The signalling receptor MCAM coordinates apical-basal polarity and planar cell polarity during morphogenesis. Nat. Commun. 8, 15279 doi: $10.1038 /$ ncomms15279 (2017).

Publisher's note: Springer Nature remains neutral with regard to jurisdictional claims in published maps and institutional affiliations.

This work is licensed under a Creative Commons Attribution 4.0 International License. The images or other third party material in this article are included in the article's Creative Commons license, unless indicated otherwise in the credit line; if the material is not included under the Creative Commons license, users will need to obtain permission from the license holder to reproduce the material. To view a copy of this license, visit http://creativecommons.org/licenses/by/4.0/

\footnotetext{
(C) The Author(s) 2017
} 